\title{
Responses of Neurons in Inferior Temporal Cortex During Memory- Guided Visual Search
}

\author{
LEONARDO CHELAZZI, ${ }^{1,2}$ JOHN DUNCAN, ${ }^{1,3}$ EARL K. MILLER, ${ }^{1,4}$ AND ROBERT DESIMONE ${ }^{1}$ \\ ${ }^{1}$ Laboratory of Neuropsychology, National Institute of Mental Health, Bethesda, Maryland 20892-4415; ${ }^{2}$ Dipartimento \\ di Scienze Neurologiche e della Visione, Sezione di Fisiologia, University of Verona, Verona 37134, Italy; ${ }^{3}$ Medical \\ Research Council Applied Psychology Unit, Cambridge CB2 2EF, United Kingdom; ${ }^{4}$ Department of Brain and \\ Cognitive Sciences and The Center for Learning and Memory, MIT, Cambridge, Massachusetts 02139
}

\begin{abstract}
Chelazzi, Leonardo, John Duncan, Earl K. Miller, and Robert Desimone. Responses of neurons in inferior temporal cortex during memory-guided visual search. J. Neurophysiol. 80: 2918-2940, 1998. A typical scene will contain many different objects, few of which are relevant to behavior at any given moment. Thus attentional mechanisms are needed to select relevant objects for visual processing and control over behavior. We examined this role of attention in the inferior temporal cortex of macaque monkeys, using a visual search paradigm. While the monkey maintained fixation, a cue stimulus was presented at the center of gaze, followed by a blank delay period. After the delay, an array of two to five choice stimuli was presented extrafoveally, and the monkey was rewarded for detecting a target stimulus matching the cue. The behavioral response was a saccadic eye movement to the target in one version of the task and a lever release in another. The array was composed of one "good" stimulus (effective in driving the cell when presented alone) and one or more "poor" stimuli (ineffective in driving the cell when presented alone). Most cells showed higher delay activity after a good stimulus used as the cue than after a poor stimulus. The baseline activity of cells was also higher preceding a good cue, if the animal expected it to occur. This activity may depend on a top-down bias in favor of cells coding the relevant stimulus. When the choice array was presented, most cells showed suppressive interactions between the stimuli as well as strong attention effects. When the choice array was presented in the contralateral visual field, most cells initially responded the same, regardless of which stimulus was the target. However, within 150-200 ms of array onset, responses were determined by the target stimulus. If the target was the good stimulus, the response to the array became equal to the response to the good stimulus presented alone. If the target was a poor stimulus, the response approached the response to that stimulus presented alone. Thus the influence of the nontarget stimulus was eliminated. These effects occurred well in advance of the behavioral response. When the array was positioned with stimuli on opposite sides of the vertical meridian, the contralateral stimulus appeared to dominate the response, and this dominant effect could not be overcome by attention. Overall, the results support a "biased competition" model of attention, according to which 1 ) objects in the visual field compete for representation in the cortex, and 2) this competition is biased in favor of the behaviorally relevant object by virtue of "top-down" feedback from structures involved in working memory.
\end{abstract}

INTRODUCTION

A typical visual scene contains many different objects, not all of which can be fully processed by the visual system at any given time. Thus attentional mechanisms are needed to limit processing to items that are currently relevant to behavior (Broadbent 1958; Bundesen 1990; Desimone and Duncan 1995; Duncan 1996; Neisser 1967; Treisman 1969; Tsotsos 1990). Mechanisms for spatially directed attention have been described not only in the dorsal stream that mediates spatial perception (e.g., Bushnell et al. 1981; Colby et al. 1996; Lynch et al. 1977; Robinson et al. 1978, 1995; Steinmetz et al. 1994; Treue and Maunsell 1996) but also in the ventral stream that mediates object recognition (Connor et al. 1996; Luck et al. 1997; Moran and Desimone 1985; Motter 1993), including areas V2, V4 and the inferior temporal (IT) cortex. When two or more stimuli are located within the receptive field (RF) of cells in V2 or V4, and the animal attends to one of them, the cell's response is predominantly determined by the attended stimulus (Luck et al. 1997; Moran and Desimone 1985). The response to the unattended stimulus may be completely blocked, even though it is an otherwise optimal sensory stimulus within the RF. Comparable effects are found in IT cortex, although the RFs of IT cells are much bigger and the attentional effects generalize over a much larger spatial range than in V2 and V4 (Moran and Desimone 1985). By contrast, more subtle and variable effects of spatially directed attention are found when only a single stimulus is located within the RF of a V2 or V4 neuron (Haenny et al. 1988; Luck et al. 1997; Maunsell et al. 1991; Moran and Desimone 1985).

We have proposed a "biased competition" model to explain the operation of spatially directed attention in ventral stream areas (Desimone 1996; Desimone and Duncan 1995; Duncan 1996; Luck et al. 1997). The model is based on the notion that objects in the visual field activate neural representations in the cortex in a parallel fashion, and that the cells participating in these representations engage in competitive interactions. Frequently, these interactions are evidenced by suppresive effects of one stimulus on the response to another (Miller et al. 1993a; Reynolds et al. 1994, 1995; Rolls and Tovee 1995; Sato 1989), and the effects are typically strongest when nearby stimuli activate nearby cells in the cortex, such as cells with similar RFs. Further, these competitive interactions are biased in favor of cells participating in one object representation versus another by many different mechanisms, including both bottom-up, or stimulus-driven mechanisms (e.g., high relative contrast) and top-down, or attentional, mechanisms (Luck et al. 1997; Moran and Desimone 1985; Reynolds and Desimone 1997; Reynolds et al. 1996). According to the model, the changes 
in baseline firing rates (Luck et al. 1997) as well as the modest increases in sensory-evoked responses found in some studies when attention is directed to the RF stimulus (e.g., Spitzer et al. 1988) are effects of the top-down bias. When two stimuli are present within the RF, an attentional bias in favor of one of them will drive the competition in favor of that stimulus, resulting in the suppression of responses to the unattended stimulus.

Although most studies of attention in the cortex have focused on spatially directed attention, nonspatial attention plays at least as important a role in behavior. Behaviorally relevant stimuli in the visual field are often found on the basis of nonspatial features such as shape and color rather than location (Duncan and Humphreys 1989; Treisman and Gelade 1980; Wolfe et al. 1989). When searching for a "face in a crowd," for example, search is guided by information about the features of the face stored in long or shortterm memory.

Recent evidence suggests that such nonspatial attentional mechanisms may also be explained by the biased competition model. Neurons in IT cortex were recorded while animals performed a visual search task in which a cue stimulus was followed, after a delay, by one or more stimuli in an array (Chelazzi et al. 1993). The animal was rewarded for making an eye movement to the target stimulus in the array that matched the cue. In an initial analysis of the results, we found that the baseline activity of cells was higher during the delay following a preferred cue, which could be due to a bias in favor of cells coding the relevant object features. Furthermore, when the array was presented, responses were determined primarily by the target stimulus; responses to nontarget stimuli were suppressed. In the present study, we have conducted a full analysis of the results from the visual search task, including the nature of the competitive bias during the delay, the time course of the attention effects, the role of sensory competition between target and distracter stimuli, and the special role of the vertical meridian (VM). We also present new results from task conditions directed at the nature of the competitive bias during the delay as well as results from a new search task that did not require an eye movement to the target. The latter task was designed to test whether the attentional effects depended on the specific motor response made to the target.

\section{METHODS}

Three adult male rhesus monkeys weighing $7.5-9 \mathrm{~kg}$ were used. The general methods were described previously (Miller et al. 1993b) and will only be briefly described here. Under aseptic conditions, a head post, recording chamber, and scleral eye coil for monitoring eye position (Robinson 1963) were implanted while the monkeys were under isofluorane anesthesia. One animal was implanted with chambers over both hemispheres, and the other two were implanted with a single chamber.

\section{Stimuli}

The stimuli consisted of a set of 24 complex, multicolored pictures presented on a computer graphics display. The stimuli ranged from $1 \times 1^{\circ}$ to $2 \times 2^{\circ}$ in size and were digitized from magazine pictures; some were of identifiable objects, and some were simply colored textures and patterns.

For most cells, we selected three stimuli from the set to use in the search tasks. These stimuli were selected for each cell while the animal performed a simple fixation task (below). One stimulus was selected on the basis of eliciting the best response from the set, and a second was selected on the basis of eliciting little or no response. For simplicity, we will refer to these as the "good" and the "poor" stimuli. A third, "neutral," stimulus was also selected without any specific response requirement, but in many cases it elicited a response that was intermediate between those elicited from the good and poor stimuli. A few cells in the saccade task were studied with a larger number of stimuli. For these cells, we selected one good stimulus and two to four poor stimuli.

\section{Saccade task}

TWO-STIMULUS ARRAYS. The basic task is schematically shown in Fig. 1. Each trial began with the presentation of a fixation target (white spot, $0.1^{\circ}$ diam) at the center of the display, which the monkey was required to fixate. After an interval of 700-1,000 ms, a cue stimulus was presented over the fixation target for $300 \mathrm{~ms}$, followed by a $1,500-\mathrm{ms}$ blank delay period. The fixation target remained on during the delays, and the animal was required to maintain fixation within a $1^{\circ}$ diam window from the beginning of the trial until the end of the 1,500-ms delay. Eye movements at any time from the onset of fixation to the end of the delay period were counted as errors, and the trial was aborted.

At the end of the delay, an array of two stimuli was presented extrafoveally. On "target-present" trials, one of the stimuli (the target) matched the previous cue, and the other (the distracter) did not. The monkey was required to make a saccade to the target within $700 \mathrm{~ms}$ of the array onset. After the monkey fixated the target for $150 \mathrm{~ms}$, the display was turned off, a drop of juice reward was given, and the trial was terminated. Eye movements to the distracter at any time were counted as errors and immediately terminated the trial. On "target-absent" trials, neither of the two stimuli in the array matched the cue. On these trials, the array was presented for $600 \mathrm{~ms}$, followed by a 1,000-ms delay period, and the monkey was required to maintain fixation on the fixation target during this entire period. At the end of the delay, a single stimulus matching the cue was presented extrafoveally, and the monkey was rewarded for making a saccade to it. Half of the trials were targetpresent trials and half were target-absent trials.

The goal of the task was to measure IT responses to a given stimulus in the array on trials when it was the attended target versus on trials when it was an irrelevant distracter. Because all of the stimuli in the array would typically be contained within a large IT RF, we adapted a technique first used by Moran and Desimone (1985) to study spatial attention in area V4 and IT cortex. In this technique, two stimuli are placed inside the RF of the recorded neuron, one of which is effective (good) in driving the cell by itself and one of which is ineffective (poor). The poor stimulus can then be treated as though it were "outside" the RF. One can then measure the effects of attention on the response to the good stimulus by comparing the firing rate in trials where the monkey attends to the good stimulus versus trials where the monkey attends to the poor stimulus.

In the present experiment, we measured the effects of attention on the response to stimulus arrays composed of the good and poor stimuli for each cell. On target-present trials, the cue at the start of the trial determined which stimulus was the target. For example, on trials when the good stimulus was the cue, the animal was rewarded for selecting the good stimulus as the target, whereas on trials when the poor stimulus was the cue, the good stimulus became behaviorally irrelevant and the animal was rewarded for selecting the poor stimulus as the target.

On target-absent trials, we measured the response to the array with the neutral stimulus for the cell as the cue. This gave a measure of the response to the good and poor stimulus paired together that 


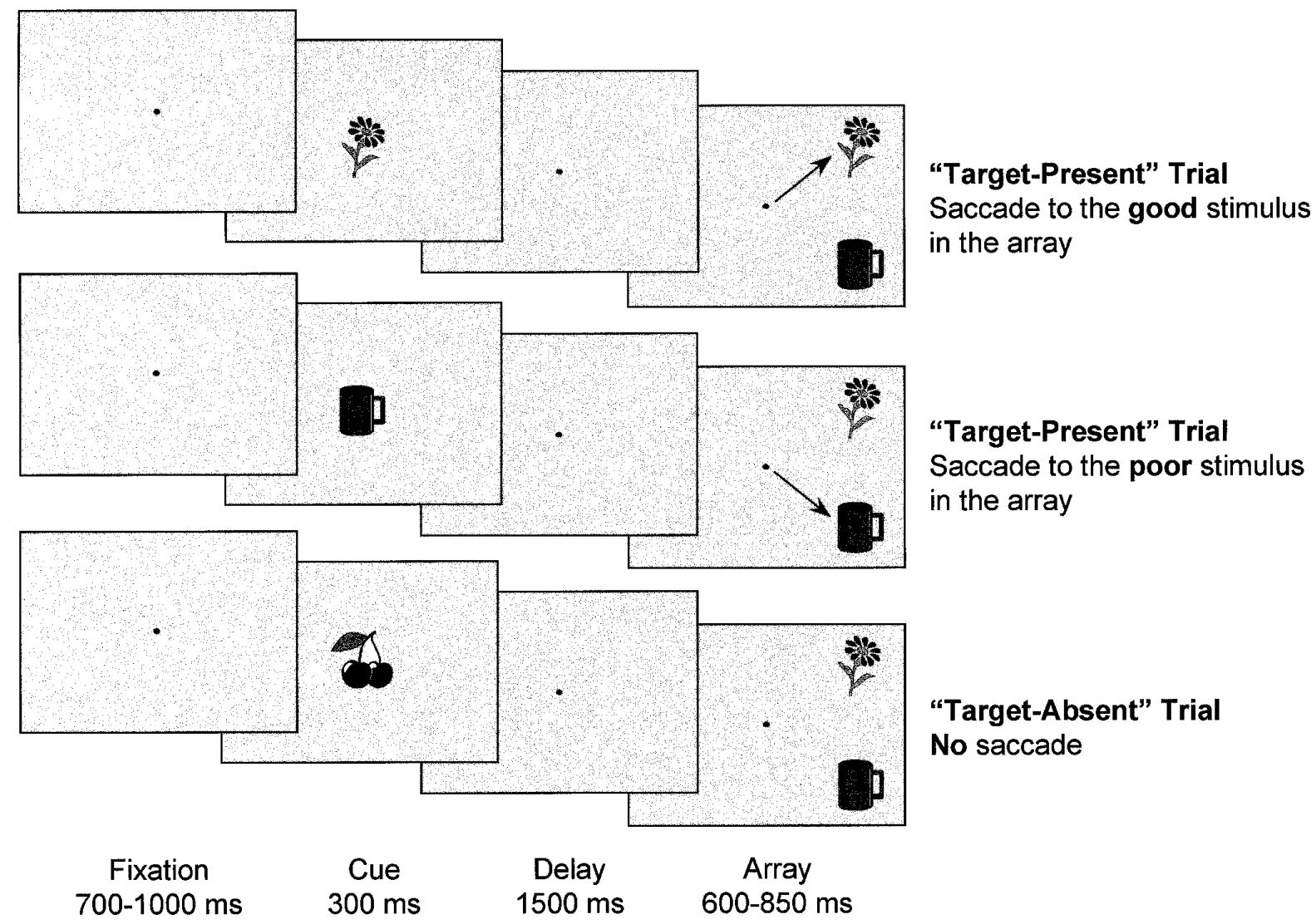

FIG. 1. Stimulus sequences for representative trials in the task with 2-stimulus arrays, with the array confined to the hemifield contralateral to the recording site.

was independent of target selection. Although the focus of the study was on the response to arrays composed of the good and poor stimulus, the trials were completely balanced so that the good, poor, and neutral stimuli appeared equally often as a cue, target, and distracter in the arrays.

Stimuli in the array were presented along an imaginary circle centered on fixation, usually at an eccentricity of $4-7^{\circ}$. The two stimuli were randomly positioned at symmetrical locations on opposite sides of either the horizontal meridian (HM) or VM of the visual field. When positioned across the HM, one was in the upper quadrant and the other in the lower quadrant of the hemifield contralateral to the recording site. When positioned across the VM, one was in the lower-right quadrant and the other in the lowerleft quadrant. Because the relative locations of the stimuli varied randomly across trials, the animal had to find the target based on its features.

ONE-STIMULUS ARRAYS. On some trials, the search array was replaced by a single stimulus, which was either the good or poor stimulus for the cell. The stimulus appeared randomly at each of the positions used for the two-stimulus arrays. The stimulus was equally often a target and a nontarget, depending on the preceding cue. These trials were treated as target-present and target-absent trials, respectively, which are described in the previous section. All other conditions of the task were the same as in the task with two-stimulus arrays.

FIXATION-ONLY TASK. On some trials, a cue stimulus was followed by a single matching or nonmatching test stimulus at the center of gaze. The cue was on for $300 \mathrm{~ms}$, the delay was 1,500 $\mathrm{ms}$, and the final stimulus was on for $300 \mathrm{~ms}$. On these trials, the animal was rewarded for simply maintaining fixation until the end of the stimulus sequence. The purpose of these trials was to test for any differences in delay activity when memory of the cue stimulus was not required.

THREE- AND FIVE-STIMULUS ARRAYS. For some cells, the search arrays were composed of either three or five stimuli. In this condition, stimuli were evenly spaced along a hemicircle within the contralateral hemifield. One of the stimuli was a good stimulus for the cell, and the remaining stimuli, all different from one another, were poor ones. All other conditions were the same as with the two-stimulus arrays, and there was an equal number of targetpresent and target-absent trials.

BLOCKING AND INTERLEAVING OF TRIALS. Cells were typically studied with 400-480 trials, which allowed for 10-12 correct trials for each trial type. For cells studied with the combination of two-stimulus arrays, one-stimulus arrays, and the fixation-only control task (i.e., excluding cells studied with 3- and 5-stimulus arrays), trials were divided among these three tasks in the ratio of $6: 3: 1$, respectively. Trials for the three different tasks were run in separate blocks. A given block typically contained 10-30 trials, and each block was typically repeated 2-3 times, randomly interleaved, during the recording of an individual cell.

All cells were studied in a "blocked cue" version of the three tasks, in which the same stimulus was used as the cue for 10-30 trials in a row. Thus, trials were blocked according to the cue, the number of stimuli in the search array (1-stimulus arrays and 2stimulus arrays), and the task (search task or fixation only).

In addition, a subset of cells was studied in a more difficult, "unblocked cue," version of the search task with two-stimulus arrays, in which the cue varied randomly from trial to trial. In this version of the task, the delay interval between offset of the cue and onset of the search array was increased to $3,000 \mathrm{~ms}$. 


\section{Lever release task}

Cells studied in the lever release task were tested using a combination of two-stimulus arrays, single-stimulus arrays, and a foveal task. However, we did not test three- or five-stimulus arrays in the lever release task.

TWO-STIMULUS ARRAYS. The search task with saccades described above required both an eye movement and the explicit localization of the target. To test whether these two factors were necessary for any neuronal effects of attention in the search task, we tested some cells in a variation of the task in which the behavioral response was a lever release rather than a saccade. The monkey grasped a lever to initiate the trial. A fixation target then appeared at the center of the display, which the monkey was required to fixate for the remainder of the trial. If the animal broke fixation, the trial was terminated. After an interval of 700-1,000 ms, a cue stimulus was presented over the fixation target for $300 \mathrm{~ms}$, followed by a 1,500-ms blank delay period. At the end of the delay, a search array was presented for $500 \mathrm{~ms}$ at the same extrafoveal locations tested in the saccade version of the task. On half the trials (targetpresent, or match, trials), the array contained a stimulus that matched the initial cue, and the monkey was rewarded for releasing the lever within $700 \mathrm{~ms}$ of array onset. On the other half of the trials (target-absent, or nonmatch, trials), neither stimulus in the array matched the initial cue, and the monkey had to hold the lever until after an additional 1,000-ms delay from array offset and successive presentation of a single matching stimulus.

ONE-STIMULUS ARRAYS. On some trials, the search array contained only a single stimulus. If the stimulus matched the previous cue, the trial was treated as a target-present trial, whereas if it did not match the previous cue, it was treated as a target-absent trial (see above).

All cells were studied with the blocked cue design, in which the same cue stimulus was used for 10-30 trials in a row before switching to another cue or another task.

\section{Data analysis}

Attentional effects were evaluated at both the single cell and population level using analyses of variance (ANOVAs) and $t$-tests. When statistical tests were conducted individually on every cell in a population, a $P<0.05$ criterion was used to evaluate whether the test was significant. In such cases, the number of cells with significant effects in the population was evaluated using the binomial theorem and was always found to be different from chance except where otherwise stated. Cells were assessed for visual responsiveness by conducting paired $t$-tests on the response to each cue stimulus in a time window from $50-300 \mathrm{~ms}$ poststimulus onset, compared with the firing rate in a $300-\mathrm{ms}$ prestimulus period. Visual selectivity was assessed by conducting an ANOVA and post hoc $t$-tests on the responses to the different cue stimuli. Population response histograms were created by averaging the responses of all neurons, with time bins of $10-50 \mathrm{~ms}$. It made virtually no difference whether the histograms were averaged from actual firing rates or from responses normalized to the peak rate; therefore the figures show the unnormalized responses.

\section{Histology}

At the conclusion of the experimental sessions, fluorescent dyes were injected through a cannula at the boundaries of the recording area. A few days later, after an overdose of pentobarbital sodium, the animals were perfused transcardially with formalin. Sections were cut every $50 \mu \mathrm{m}$, stained with thionin, and examined for electrode tracks and dye marks. Although older tracks could not be visualized, recording sites could be inferred from the identifiable tracks and the location of the dye marks.

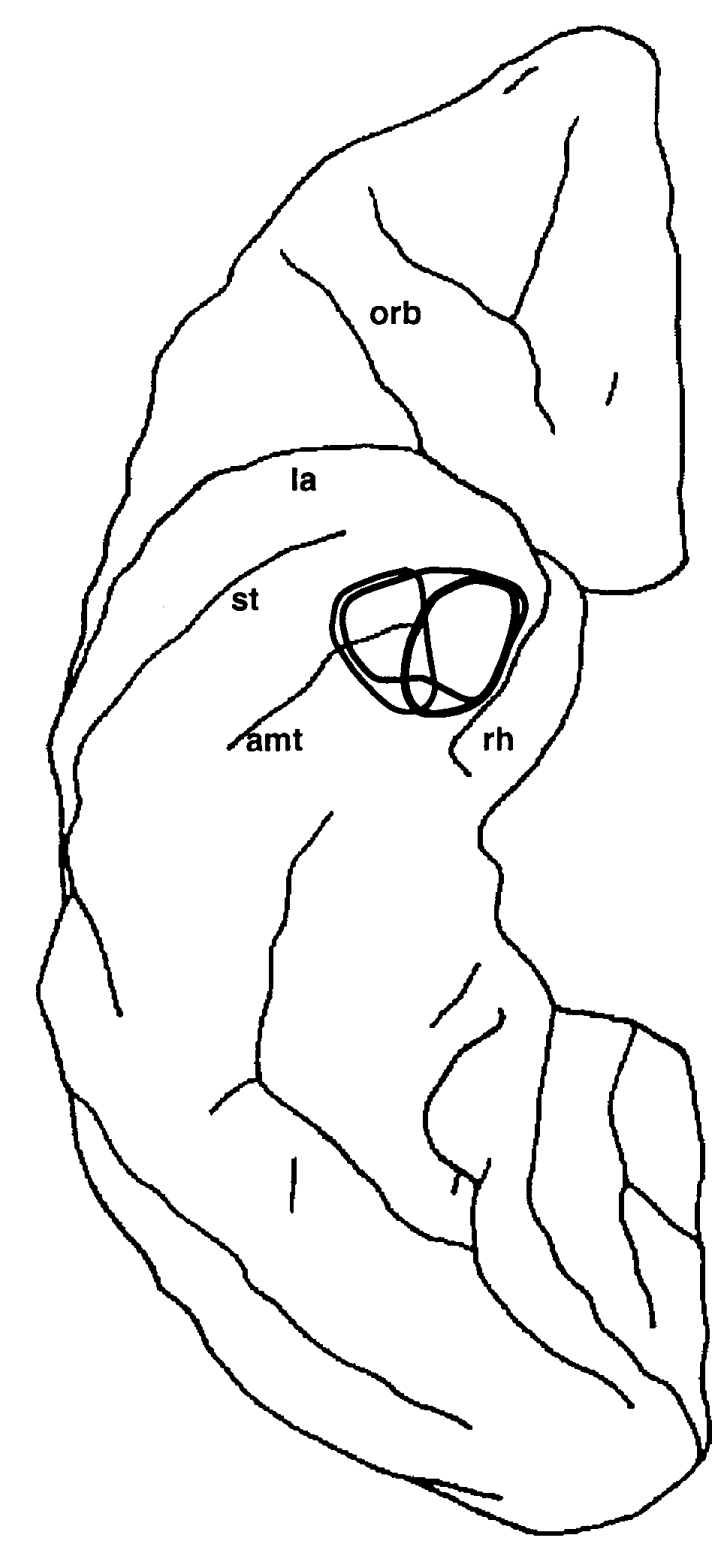

FIG. 2. Location of recording regions (closed curves) in the ventral temporal cortex of the 3 monkeys. The regions containing the recording sites were marked on ventral reconstructions of the hemispheres in each of the 3 monkeys and were then transferred to a standard ventral view. All sites were either on the ventral convexity of the temporal cortex or in the banks of the anterior middle temporal sulcus. amt, anterior middle temporal sulcus; rh, rhinal sulcus; st, superior temporal sulcus; la, lateral sulcus; orb, orbitofrontal suclus.

\section{RESULTS}

Fixation errors (before the presentation of the choice stimuli) were made on fewer than $10 \%$ of the trials, and these trials were excluded from the following performance scores. In the saccade version of the task, the animals made a saccade to the correct stimulus on 86 and $84 \%$ of the trials, with two- and five-stimulus choice arrays, respectively. On target-absent trials of this task, the animals inappropriately made a saccadic eye movement to one of the nontarget stimuli on almost $40 \%$ of the trials. Performance on the barrelease version of the task was $82 \%$ correct.

As shown in Fig. 2, the recording sites were located in 
the anterior portion of IT cortex. The sites extended from the lateral lip of the rhinal fissure, across the inferior temporal gyrus (including the perirhinal region), and onto the adjacent portion of the middle temporal gyrus. A total of 236 cells was recorded from 4 hemispheres of 3 monkeys. Of these, 42 did not have a significant response to any stimulus, and these cells will not be considered further. Eleven cells had only inhibitory responses, and these cells were also excluded from the analyses. Nearly all of the analyses required that a given cell respond differentially to the two stimuli chosen to be the good stimulus and the poor stimulus for that cell. Twenty-two cells failed to show stimulus selectivity according to an ANOVA calculated on the stimulusevoked responses, and, unless indicated otherwise, these nonselective cells were also excluded from the analyses described below. Thus there remained 161 cells with excitatory responses and stimulus selectivity, and these cells will be the focus of the remainder of the RESULTS. Of these cells, 100 were from 2 monkeys studied in the saccade task, and 61 were from the 3rd monkey studied in the lever release task.

\section{Search task with saccades (1- and 2-stimulus arrays): cue-related activity}

The results from 83 stimulus-selective cells were analyzed in the search task with saccades (an additional 17 stimulusselective cells studied with 3- and 5-stimulus arrays will be described in a later section). All of them were studied in the blocked cue design, and 28 of the 83 cells were also studied in the random cue design, in which the cue varied randomly from trial to trial.

RESPONSES TO THE CUES AND IN THE SUBSEQUENT DELAY PERIOD. As expected, the responses to the good cue in the search task were invariably greater than to the poor cue. In addition, many cells had higher maintained activity in the blank delay period after the offset of the good cue than in the delay after the offset of the poor cue. This maintenance of cue-specific activity throughout the delay period could be evidence of the "bias" in favor of the cells representing the target stimulus, as predicted by the biased competition model. A related possibility is that the activity during the delay is part of the mechanism for maintaining the memory of the cue.

Figure 3 shows histograms for the cue response and delay activity for a cell studied in both the random and blocked cue design, and Fig. 4 shows responses averaged across the entire population of cells, for the same two conditions. All of the histograms show that activity in the delays was higher on trials when the good stimulus was used as the cue for the recorded cell than when the poor stimulus was used as the cue. The cue-specific activity persisted throughout the entire delay, which was $3 \mathrm{~s}$ long in the random cue design. In the blocked cue design, but not in the random design, the activity was also higher preceding the onset of the good cue than preceding the poor cue, and this activity difference will be considered in a later section.

To assess differential delay activity across the population, we computed a $t$-test on the firing rates in the last $500 \mathrm{~ms}$ of the delay period following the good cue compared with the firing rates in the delay following the poor cue for each cell. For a large fraction $(11 / 28)$ of cells studied in the random cue design, the average delay activity following the good cue ( 6.8 spikes/s) was significantly higher than following the poor cue ( 4.0 spikes/s). Similarly, most cells (43/ 83 ) studied in the blocked design had significantly higher activity in the delay following the good cue (7.9 spikes/s) than in the delay following the poor cue $(5.6$ spikes/s). For a few $(6 / 83)$ cells, however, the average firing rate was significantly higher following the poor cue $(8.0$ spikes/s $)$ than following the good cue $(6.0$ spikes/s).

To assess the magnitude of differential delay activity across the population, a delay activity index (DAI) was computed for all cells according to the following formula: DAI $=($ Activity following Good Cue - Activity following Poor Cue)/(Activity following Good Cue + Activity following Poor Cue).

Index values close to 1 indicate that delay activity was much higher following the good cue than following the poor cue. Values close to 0 indicate little or no difference, and values $<0$ indicate that the delay activity following the good cue was lower than the activity following the poor cue. The frequency distribution histogram of this index, shown in Fig. $5 A$ for the blocked design, is strongly shifted toward the right, indicating that most cells showed the baseline shift effect. The mean index across all 83 cells studied in the blocked design and across all 28 cells studied in the random design was 0.12 and 0.13 , respectively, corresponding to a mean increase in baseline firing rate of 28.2 and $29.2 \%$.

We noticed that some cells with strong differential delay activity following the good and poor cues were also highly stimulus selective. To test for a relationship between stimulus selectivity and delay activity across the population, we compared the DAI (described previously) for each cell with an index of stimulus selectivity. A stimulus selectivity index (SSI) was computed according to the following formula: SSI $=($ Response to Good Cue - Response to Poor Cue $) /$ (Response to Good Cue + Response to Poor Cue).

Index values close to 1 indicate high stimulus selectivity, whereas values close to 0 indicate poor selectivity. The delay index is plotted against the selectivity index in Fig. 5B. For comparison, the figure also shows separately the indexes for the 18 cells that were studied for delay activity but were excluded from all other analyses because they did not display statistically significant stimulus selectivity for the cues. For cells studied in the blocked design, there was a significant positive correlation between the two measures $(r=0.53$; $P<0.001)$ whether or not we included cells without significant stimulus selectivity. Similar results were obtained with the random cue paradigm. Thus there was a tendency for the cells with the greatest stimulus selectivity to also show the greatest difference in delay activity following the good versus the poor cue. This suggests that the feedback bias responsible for the delay activity preferentially targeted cells that best discriminated the different cue stimuli.

ACTIVITY PRECEDING ONSET OF THE CUE. Although we interpreted the higher maintained activity in the delay following the good cue as evidence for a bias in favor of cells coding the cue-target stimulus, we also considered whether it might be simply a long-lasting aftereffect of the response to the good cue. Two lines of evidence argue strongly against 


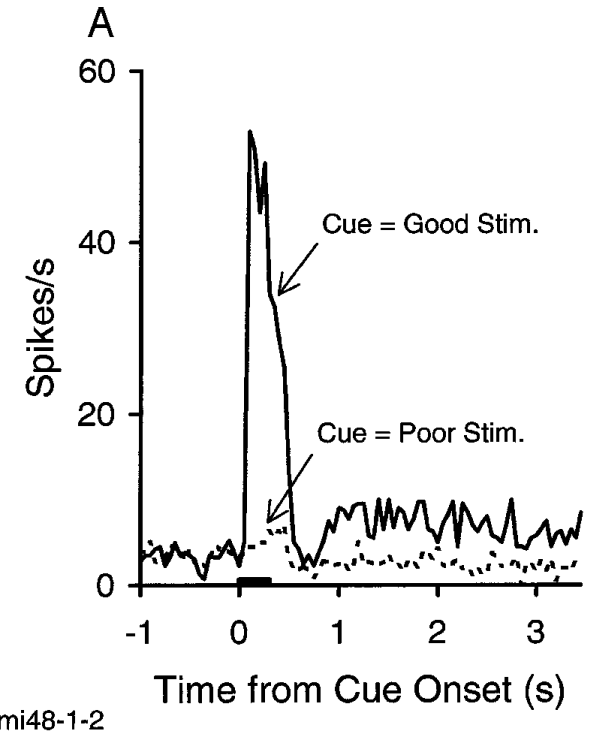

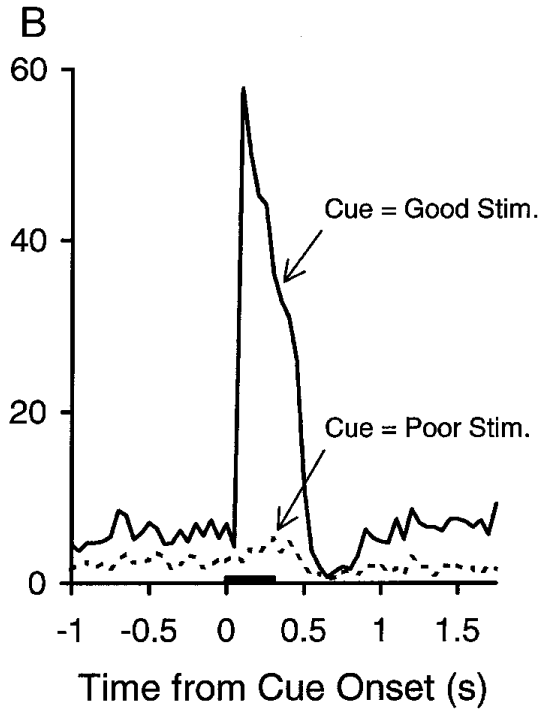

FIG. 3. Responses to the cue stimuli by an individual neuron studied in both the randomcue $(A)$ and blocked-cue $(B)$ design. The horizontal bar indicates the $300-\mathrm{ms}$ duration of the cue stimulus. Binwidth is $50 \mathrm{~ms}$. this. One line of evidence comes from the fixation-only trials, which are discussed in the next section. The other evidence is that for cells studied in the blocked design, the higher maintained activity following the good cue was paralleled by a similar increase in maintained activity preceding the onset of the good cue. This can be clearly seen in the single cell and population histograms of Figs. 3 and 4. In these figures, cells studied in the blocked design had higher maintained activity both preceding and following the good cue, whereas the cells studied in the random cue design had higher activity only in the interval following the good cue. The increase in activity preceding the good cue in the blocked design could not be an artifact of nonspecific changes in cell activity across the session because the blocks of trials with the good and poor cue were interleaved. We also considered the possibility that the higher activity preceding the good cue in the block design may have been due to a lingering response to the good stimulus as target on the previous trial. To test for such lingering responses, we examined the activity preceding the cue in the random design, comparing the precue activity on trials immediately after a trial with the good stimulus as the target with the precue activity on trials immediately after a trial with the poor stimulus as target. There was no difference in activity across the two types of trials, ruling out the possibility that the activity preceding the cue was due to a lingering response to the target. Thus the maintained activity preceding the cue in the blocked design was apparently a purely "cognitive" phenomenon related to expectation of a specific cue and could not be a sensory response.

FIXATION-ONLY TRIALS. Fixation-only trials were included in the saccade task as a control condition. These trials provided a second line of evidence against the possibility that the cue-specific activity before and after the cue in the saccade task was some type of sensory phenomenon. On these trials, the cue stimulus was followed, after a 1,500 ms delay, by a matching or nonmatching stimulus at the center of gaze. The animal was rewarded for simply maintaining fixation throughout the trial. As in the blocked cue design for the saccade trials, the particular cue stimulus used at the start
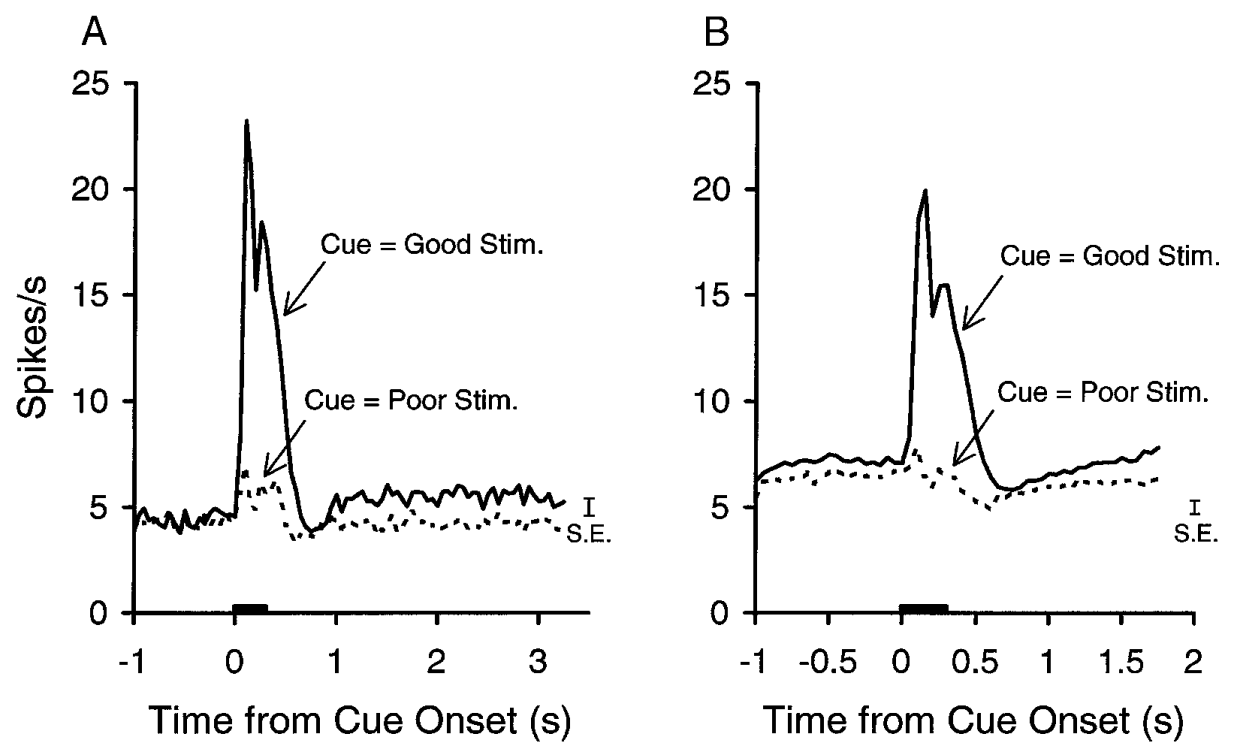

FIG. 4. Population histograms showing responses to the cue stimuli. $A$ : average from 28 cells studied in the random-cue design. $B$ : average from 83 cells studied in the blockedcue design. Horizontal bar indicates duration of the cue stimulus $(300 \mathrm{~ms})$. In this and all subsequent figures, SE is the standard error of the mean firing rate in the population, averaged from the standard error of the mean in all individual bins. Binwidth is $50 \mathrm{~ms}$. 


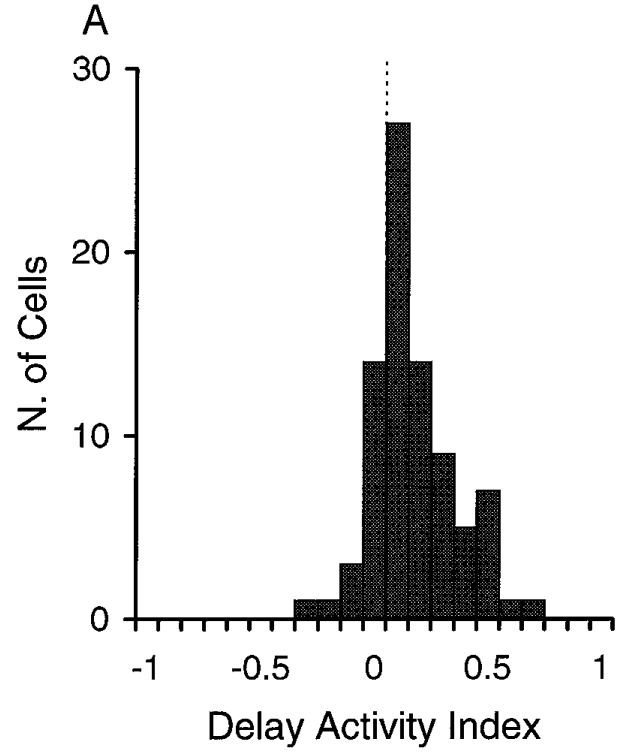

of the trial was kept constant for a block of trials. Thus the sensory conditions both before and after the cue (until the end of the delay) were identical to those of the saccade trials, but the cue had no behavioral relevance for the animal. All of the cue-specific activity that had been found both preceding and following the cue in the saccade task was eliminated in this condition. Thus the cue-specific shifts in baseline firing rates found in the saccade task must have been caused by the animal actively using the cues in the task.

Search task with saccades (1- and 2-stimulus arrays): arrays in contralateral field

A second major goal of the study was to test for a role of IT neurons in selecting the target stimulus out of the search array. Of the 83 cells studied in the blocked cue design, 58 gave a significant response to at least 1 of the 2 stimulus array configurations tested, as did 22 of the 28 cells studied in the random design. The analyses of responses to the search array were restricted to these cells.

EFFECTS OF TARGET SELECTION ON TWO-STIMULUS ARRAYS. Because the results differed depending on whether the stimuli were located in the contralateral or ipsilateral field, we will present the contralateral field data first. In this configuration, one stimulus was in the upper quadrant and one in the lower quadrant, and we pooled the data across the two possible spatial configurations of the good and poor stimuli. For simplicity, we will focus on the data in the blocked cue design, which were very similar to the data from the random cue design.

Figure 6 shows the responses of an individual cell to the search array on trials in which the target was the good stimulus or the poor stimulus, and Fig. 7 shows responses to the same comparison conditions averaged across all of the cells. The sensory conditions in the array were identical across this comparison; what varied was the target, which was determined by the cue shown at the start of the trial. The location of the target (upper vs. lower quadrant) varied randomly from trial to trial.
The population histograms of Fig. $7 A$ indicate that the initial response to the onset of the array was only slightly affected by whether the target was the good or poor stimulus for the cell. The firing rate was slightly higher in the early phase of the response on trials when the good stimulus was the target, but this appeared as though it might be a continuation of the elevated firing during the delay interval on these trials, described in the previous section.

In contrast to the small effects of target selection on the initial response to the array, the population average response began to change markedly $\sim 150-200 \mathrm{~ms}$ after array onset, depending on whether the target was the good or poor stimulus for the cell. If the target was the good stimulus, the firing rate remained high, whereas if the target was the poor stimulus, the firing rate was strongly suppressed. This can also be clearly seen in the single-cell example shown in Fig. $6 A$. Thus, consistent with the biased competition model, IT cells selective for any stimulus in the array were initially activated in parallel, regardless of which stimulus in the array was the target. After a short period of processing, responses to the good stimulus were suppressed when the poor stimulus was the target, as though the good stimulus had been filtered out of the RF. We will refer to this effect of target selection as the "target effect."

To determine the time at which the population response to the good stimulus became suppressed when the poor stimulus was the target, we computed a paired $t$-test (evaluated at $P<0.05$ ) on each $10-\mathrm{ms}$ bin in the population histograms. The onset of suppression was defined to begin at the first of two consecutive bins that showed a significant difference in response on the good and poor target trials. According to this analysis, the response to the good stimulus became significantly suppressed at $170-180 \mathrm{~ms}$ after array onset when the poor stimulus was the target.

The histograms time locked to the array onset in Fig. $7 \mathrm{~A}$ also show the average time of the saccadic eye movement, which occurred $307 \mathrm{~ms}$ after the onset of the array. This time was well after the beginning of the target effect on neuronal responses. This is shown more clearly in Figs. $6 B$ and $7 B$ in which we recomputed the histograms for both the 


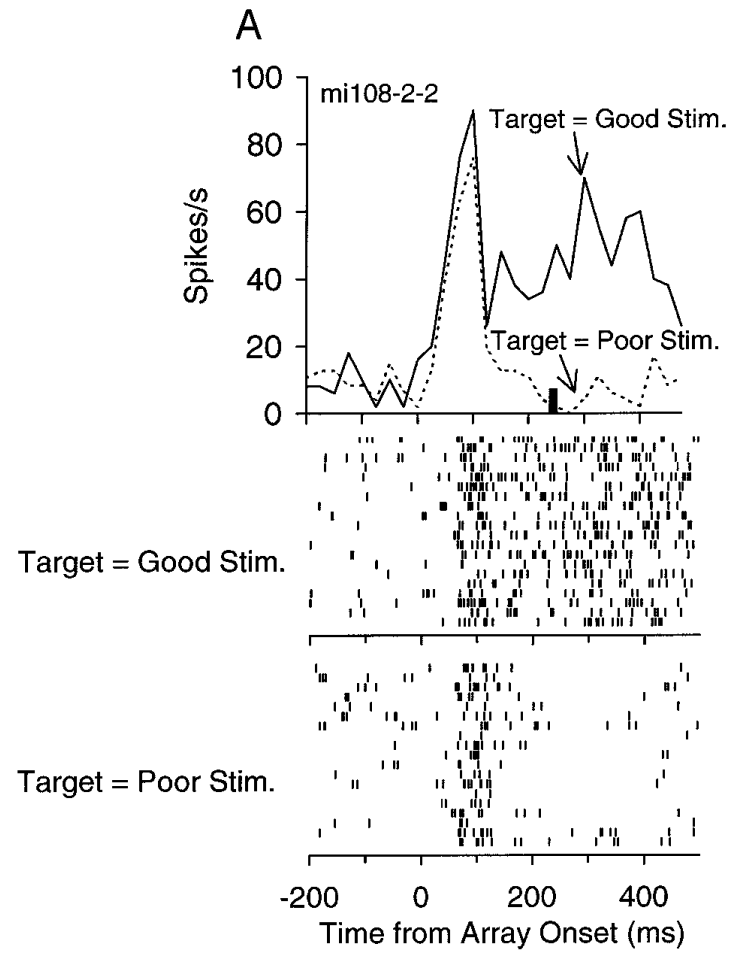

B

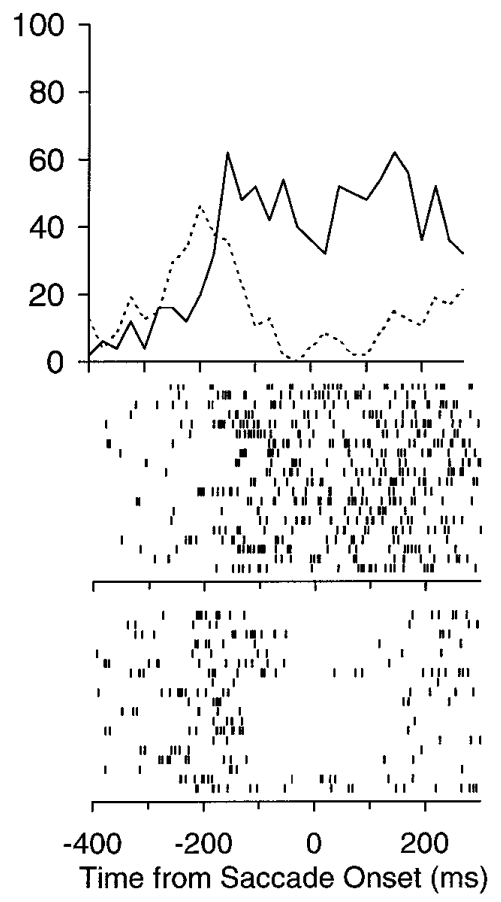

FIG. 6. Response of an individual neuron to the 2-stimulus array in the contralateral hemifield. $A$ : responses time locked to the onset of the array. Vertical bar indicates average saccadic latency to the target. $B$ : responses time locked to the onset of the saccade. Binwidth is $25 \mathrm{~ms}$. Below the histograms in $A$ and $B$ are rasters from the good-target and poor-target trials. Each tick in the rasters represents an action potential from the neuron, and each row corresponds to a different trial. individual cell example and the population, time locked to the saccadic eye movement rather than to the onset of the array. We then repeated the paired $t$-tests on each $10-\mathrm{ms}$ bin in the population histograms. These tests indicated that a significant target effect on neuronal responses began 70-80 ms before the eye movement to the target.

To explore this difference between the early and late phase of the response across cells in the population, we computed for each cell a target effect index (TEI) in two separate time windows, an early one spanning 70-170 ms after stimulus onset and a late window in the $100 \mathrm{~ms}$ before the saccade. The index was computed according to the following formula: TEI $=($ Activity on Good-Target Trials - Activity on Poor-
Target Trials)/(Activity on Good-Target Trials + Activity in Poor Target Trials ).

Figure $8 A$ shows a scatter plot of TEI in the early time window plotted against the TEI in the late window, for each cell. Most points in the plot fall to the left of the diagonal, indicating that for most cells the effect of target selection on responses increased from the early to the late window. There were, however, some individual cells that appeared to show either positive or negative target effects on responses in the early time window. In the late window, the large majority of cells $(48 / 58)$ had a positive target effect. The mean value of the index was 0.14 in the early window and 0.26 in the late window, which was a significant difference

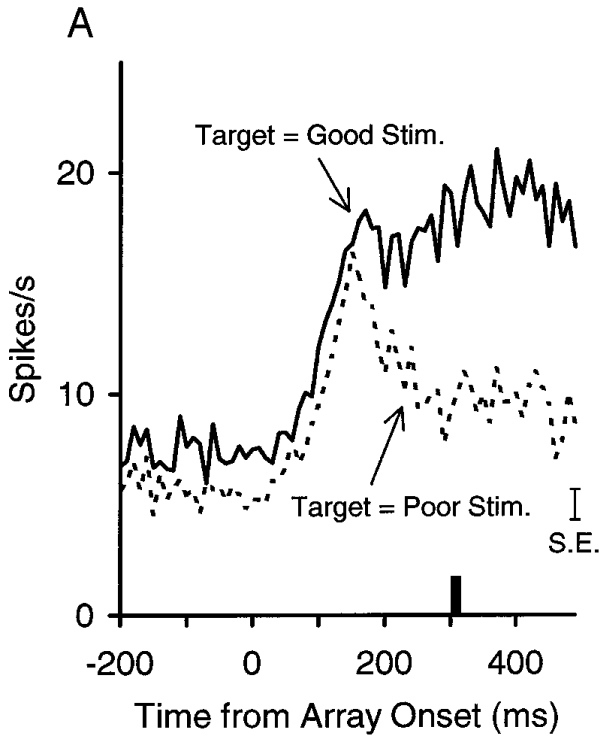

\section{B}

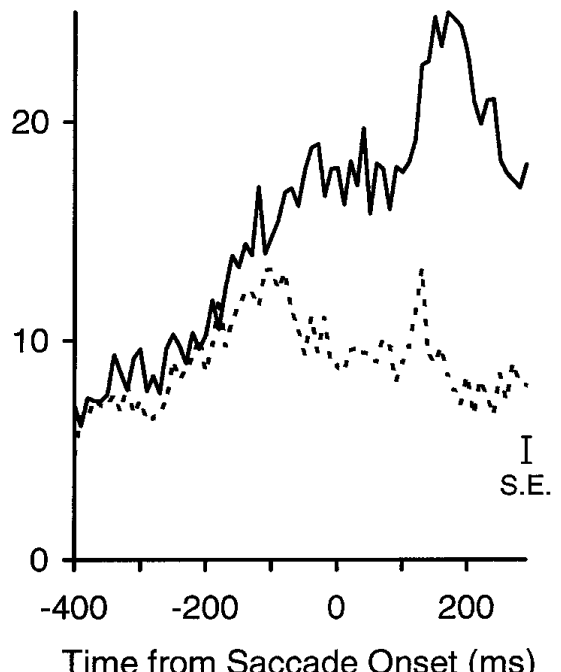

FIG. 7. Population histograms showing the average response of 58 neurons to the 2stimulus search array confined to the contralateral hemifield. $A$ : responses time locked to array onset. Vertical bar indicates average latency of the saccade to the $\operatorname{target} B$ : responses time locked to eye movement onset. Binwidth is $10 \mathrm{~ms}$. 

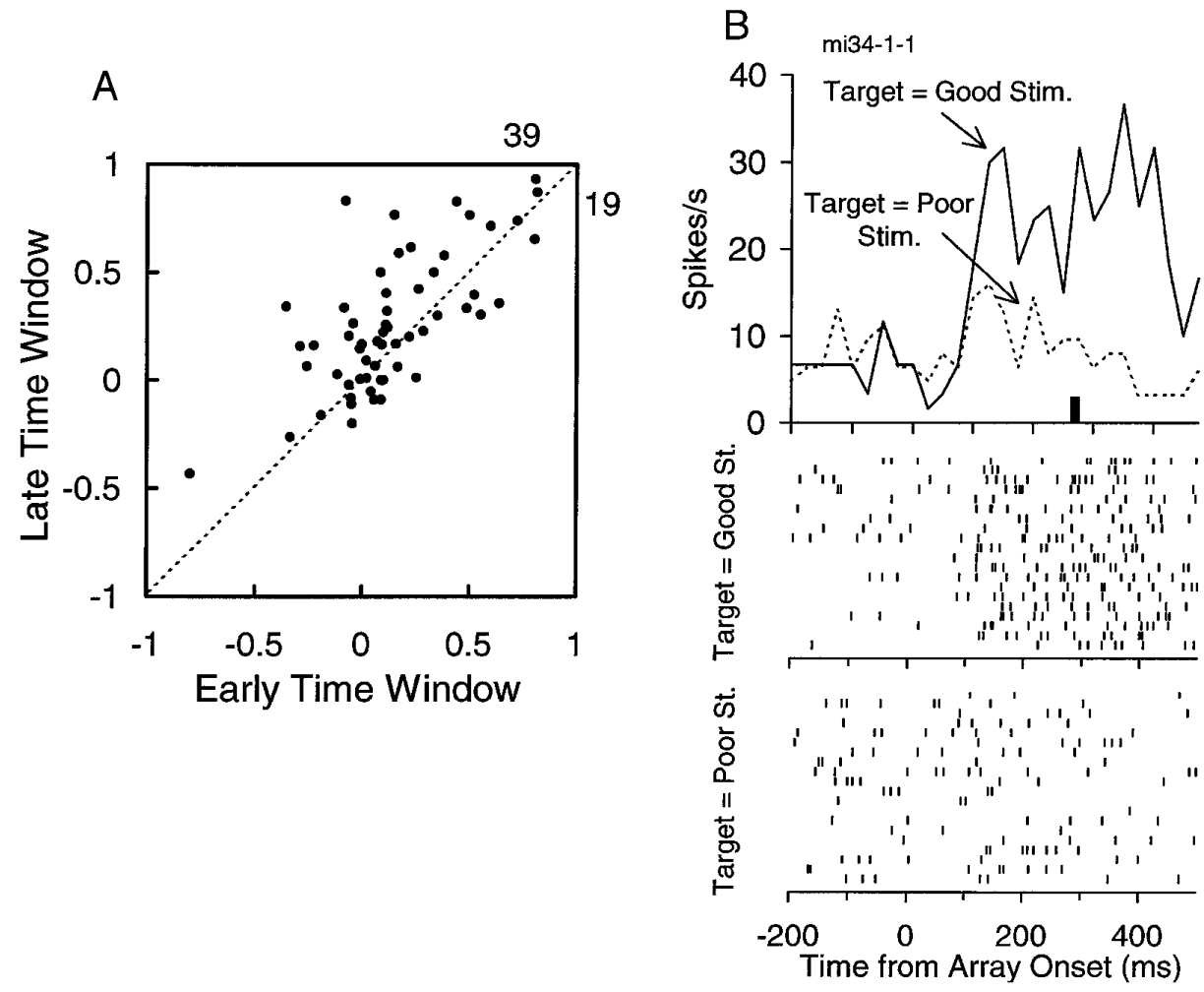

FIG. 8. $A$ : relationship between the target effect index (TEI) computed in the early time window (70-170 ms postarray onset) and the TEI computed in the late time window (last $100 \mathrm{~ms}$ before saccade onset). Numbers at the 2 sides of the top right corner of the box indicate the relative number of cells falling to the right and left of the diagonal $(---)$. $B$ : histograms showing the response of an individual cell to the search array confined to the contralateral hemifield. Responses are time locked to onset of the search array, and the vertical bar indicates the average saccadic latency to the target. Binwidth is $25 \mathrm{~ms}$. Below the histograms are rasters from the good-target and poor-target trials.

(paired $t$-test, $P<0.001$ ). The mean TEI in the late window corresponded to a percentage change in firing rate of $70.3 \%$, or a $170.4 \%$ change in the magnitude of the sensory evoked response with the baseline activity subtracted (averaging the baseline across trials with the good and poor cue as target).

To test for significant effects of target selection on the responses of individual cells, we computed a $t$-test on responses to the search array with the good versus poor stimulus as the target. The test was computed separately on responses in the early and late time windows. In the early window, 12/58 cells had a significantly larger response on trials with the good stimulus as the target versus trials with the poor stimulus as the target, compared with 3 cells that showed a significant difference in the opposite direction. By contrast, in the late window, 25/58 cells had a significantly larger response on trials with the good stimulus as target, compared with 1 cell with a significant difference in the opposite direction ( the number of cells with significant positive and negative target effects were 20 and 0 , respectively, when significance was assessed at the $P<0.01$ level). This proportion of cells with significant positive effects increased to $34 / 58$ when the analysis window was lengthened to include up to $50 \mathrm{~ms}$ beyond the initiation of the saccade (presumably before any IT neuron could respond to the target stimulus shifted to the fovea). These results, which are consistent with the distribution of the TEI, demonstrate that a few cells do show significant effects of target selection on their response in the early time window but that there is a substantial increase in the incidence of significant effects in the late window. Figure $8 B$ shows an example of a cell that showed a significant target effect in the early time window.

The data from the cells studied with the random cue design were very similar to those obtained in the blocked cue de- sign, including the development of target effects over time. Indeed, in the random cue design the effects of target selection in the early window were, if anything, smaller than those found in the blocked cue design, whereas the effects in the late window were at least as large as those in the blocked cue design.

EFFECTS OF TARGET SELECTION ON ONE-STIMULUS ARRAYS. To help interpret the results with two stimuli inside the RF, we examined the effects of target selection with only a single stimulus inside the RF. Competition between the good and poor stimulus was obviously eliminated when there was only a single stimulus inside the RF. In target-present trials, the test stimulus matched the previous cue, and the animal was rewarded for making a saccade to it. In target-absent trials the test stimulus did not match the previous cue, and the monkey was required to simply maintain fixation until a third, matching, stimulus appeared at the end of the trial ( see METHODS). To assess the effects of selection on the response to an individual stimulus inside the RF, we therefore compared the response to the good and poor stimuli presented alone on target-present trials with the response to the same stimuli presented alone on target-absent trials.

Figure $9 A$ shows the population histograms for the good and poor stimuli presented alone, in both the target-present and target-absent trials. On average, the response to the stimuli was very similar on target-present and target-absent trials, until the time of the eye movement on target-present trials. The latency of the eye movement was $228 \mathrm{~ms}$, which was significantly $(P<0.001)$ faster than on the trials with twostimulus arrays $(307 \mathrm{~ms})$. The eye movement then moved the image of the target stimulus onto the center of gaze, which clearly changed the response. Thus, when competition 


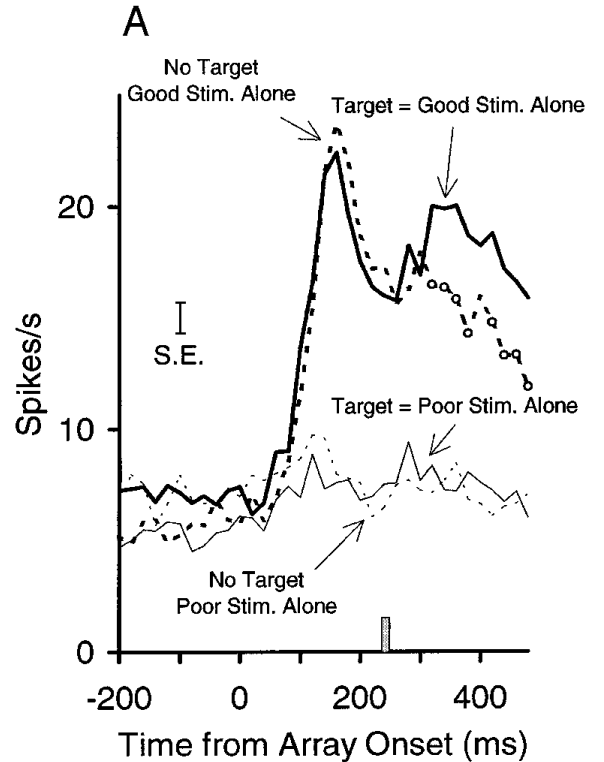

B

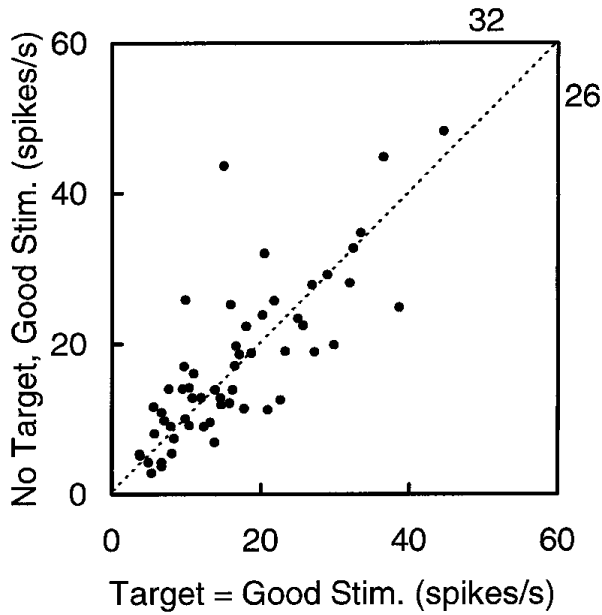

FIG. 9. A: population histograms averaged from 58 cells showing the response to the good and poor stimuli presented alone, both in target-present and target-absent trials. Stippled vertical bar indicates average saccadic latency in target-present trials. Empty circles on the "No Target, Good Stim. Alone" curve indicate bins in which the response differed significantly from that in the "Target $=$ Good Stim. Alone" condition. Binwidth is $20 \mathrm{~ms}$. $B$ : relationship between response to the good stimulus alone in targetpresent and target-absent trials. Numbers at the top right corner of the plot indicate relative number of cells falling to the right and to the left of the diagonal $(---)$. between stimuli in the RF was eliminated, the effects of target selection on responses were dramatically reduced.

The scatter plot in Fig. $9 B$ shows the responses to the good stimulus on target-present and target-absent trials for each cell individually, measured in a time window between 120 and $220 \mathrm{~ms}$ after stimulus onset. Consistent with the population histogram, the response to the good stimulus on target-present trials was similar to the response on targetabsent trials for most cells, although a few cells did show either a somewhat larger or smaller response to the good stimulus when it was a target. We also computed a $t$-test on the response to the good stimulus in target-present and target-absent trials for each cell (using the same time window between 120 and $220 \mathrm{~ms}$ after stimulus onset). According to this test, only a few cells (9/58) gave a significantly larger response on target-present trials versus target-absent trials, and an equal number of cells (9/58) showed significant effects in the opposite direction. Again, these results indicate that selecting a single stimulus as a target does not consistently cause an enhanced response to that stimulus across the population. Much larger effects of target selection are found when two stimuli compete within the RF.

SENSORY INTERACTIONS BETWEEN GOOD AND POOR STIMULI. Once we established that the effects of target selection were much larger when two stimuli competed within the RF, we next examined the nature of that competition. The first step was to determine the sensory response to the good stimulus alone, the poor stimulus alone, and the combined good and poor stimulus in the array. It would have been optimal to obtain these sensory responses in the absence of both target selection and attention. Unfortunately, it is not possible to require monkeys to attend to "nothing" while stimuli are presented. However, the target-absent trials afforded the opportunity to measure responses to the different sensory conditions in the array with target selection eliminated as a factor and attention presumably held constant. Trials in which the third, neutral, stimulus appeared as a cue were used to obtain the sensory response to the array composed of both the good and the poor stimulus (see Fig. 1). Al- though the monkey must have attended to the array, there was no basis for the monkey to attend selectively to one stimulus rather than the other, because neither stimulus was a target. Only the data from the blocked cue design were used for these analyses because not all trial types were run in the random cue design.

The histograms in Fig. $10 \mathrm{~A}$ show that, on average, the poor stimulus had a suppressive effect on the response to the good stimulus, because the response to the two-stimulus array was intermediate between the response to the good stimulus alone and the response to the poor stimulus alone, all on target-absent trials. Similarly, the response to the twostimulus array on target-absent trials was also intermediate between the response to the same array when the good stimulus was the target and when the poor stimulus was the target on target-present trials (Fig. 10B). Figure 11 shows scatter plots of responses to the good or poor stimulus alone versus the response to the two-stimulus array in the target-absent condition for all cells. For some cells, the response to the two-stimulus array was equal to the response to the good stimulus alone (points along the diagonal in Fig. 11A). However, for most cells the response to the array was smaller than the response to the good stimulus alone and, in some cases, was even reduced to the level of response to the poor stimulus presented alone. For only $3 / 58$ cells did the response to the 2 -stimulus array fall significantly outside the range of responses to the good or poor stimulus alone, according to $t$-tests calculated over a time interval 200-300 ms after array onset. Together, the results support the notion of a competitive interaction between the good and the poor stimulus in the array.

EFFECT OF TARGET SELECTION ON SENSORY INTERACTIONS. The next step was to ask how target selection affected the sensory interactions (described in the previous section) between the good and poor stimuli in the array. To this aim, we compared the responses to 1 ) the two-stimulus array with the good stimulus as the target, 2) the two-stimulus array with the poor stimulus as the target, 3 ) the good stimulus presented alone, and 4) the poor stimulus presented alone. 

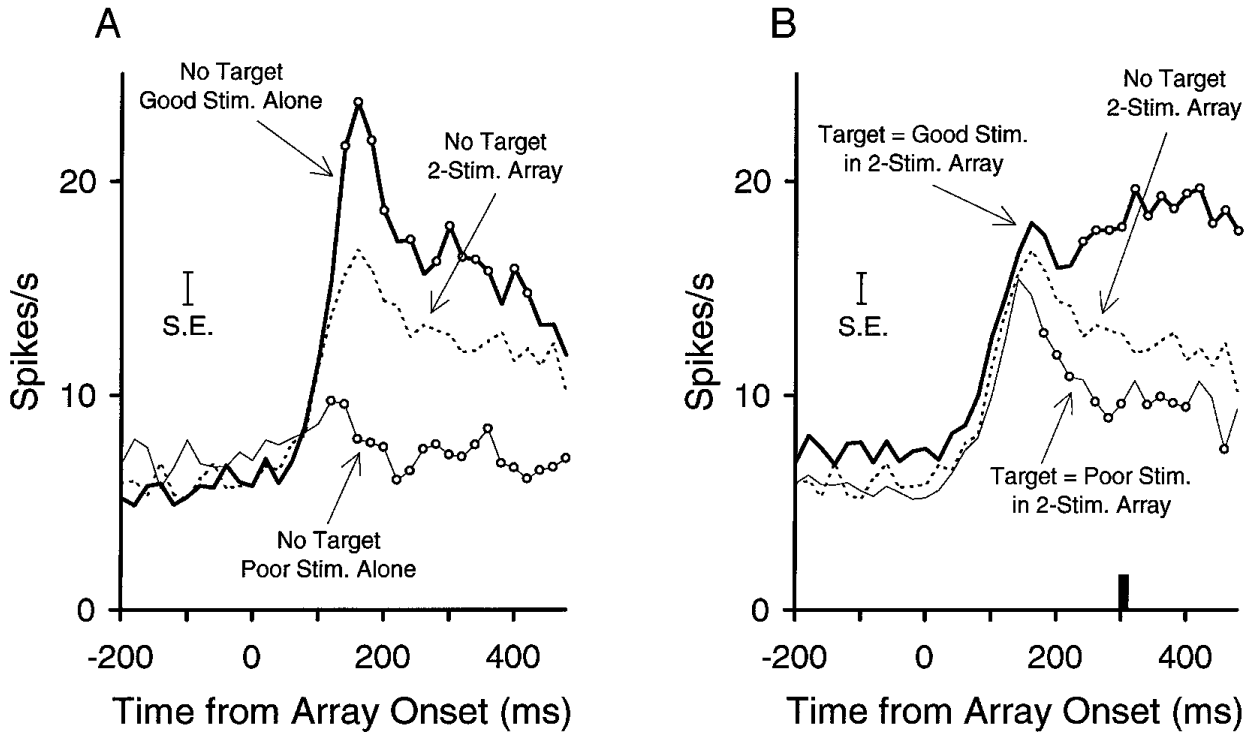

FIG. 10. A: population histograms averaged from 58 cells. Responses to the good and poor stimuli presented alone are compared with the response to the search array. In all 3 conditions responses were measured in target-absent trials. Empty circles on the "No Target, Good Stim. Alone" curve indicate bins in which the response differed significantly from that in the "No Target, 2-Stim. Array" condition. Empty circles on the "No Target, Poor Stim. Alone" curve indicate bins in which the response differed significantly from that in the "No Target, 2-Stim. Array" condition. $B$ : population histograms averaged from 58 cells. Responses to the search array when the good or poor stimulus was the target are compared with responses to the same array in target-absent trials. Empty circles on the "Target = Good Stim. in 2-Stim. Array" curve indicate bins in which the response differed significantly from that in the "No Target, 2-Stim. Array" condition. Empty circles on the "Target $=$ Poor Stim. in 2-Stim. Array" curve indicate bins in which the response differed significantly from that in the "No Target, 2-Stim. Array" condition. Binwidth is $20 \mathrm{~ms}$.

For the latter two responses, we used the data from the onestimulus arrays in target-present trials, although as indicated above, the responses on these trials were the same as on the target-absent trials.

Figure $12 \mathrm{~A}$ shows the population histograms for the four conditions, time locked to stimulus onset, and Fig. $12 \mathrm{~B}$ shows the same data time locked to the eye movement. The initial response to the two-stimulus array with the good stimulus as target was smaller than the response to the good stimulus alone. This was presumably because the poor stimulus in the array had an overall suppressive effect on the response to the good stimulus in the array (see Figs. $10 \mathrm{~A}$ and 11 , described in the previous section). However, by 200 ms after stimulus onset, the response to the two-stimulus array with the good stimulus as target equaled the response to the good stimulus presented alone. That is, the suppressive influence of the poor stimulus was eliminated in the later phase of the response. Cells responded as though the poor stimulus had been filtered out of the RF.

Conversely, the initial response to the two-stimulus array with the poor stimulus as target was larger than the response to the poor stimulus presented alone. This was presumably because the cells were initially responding to the presence of the good stimulus in the array and RF. However, by 250 $300 \mathrm{~ms}$ after stimulus onset, the response to the array with the poor stimulus as target closely approached the response
A

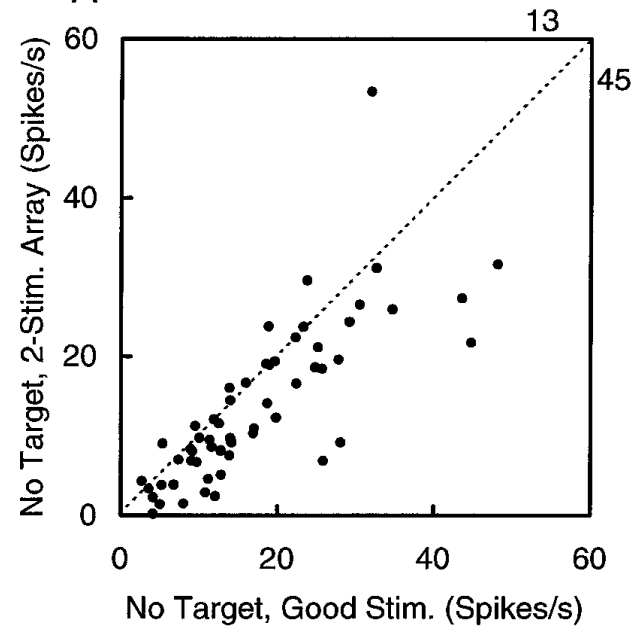

B

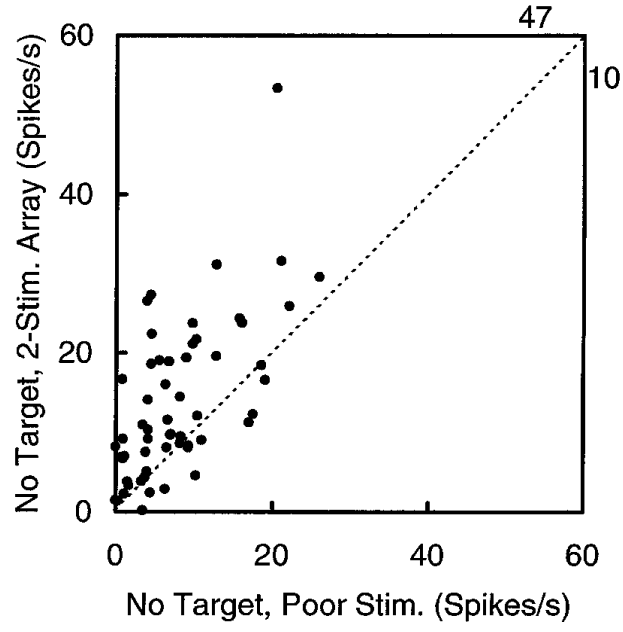

FIG. 11. A: relationship between the response to the good stimulus presented alone and the response to the 2-stimulus array. $B$ : relationship between the response to the poor stimulus presented alone and the response to the 2-stimulus array. In both plots, figures near the top right corner indicate the number of cells falling to the right and left of the diagonal $(---)$. 


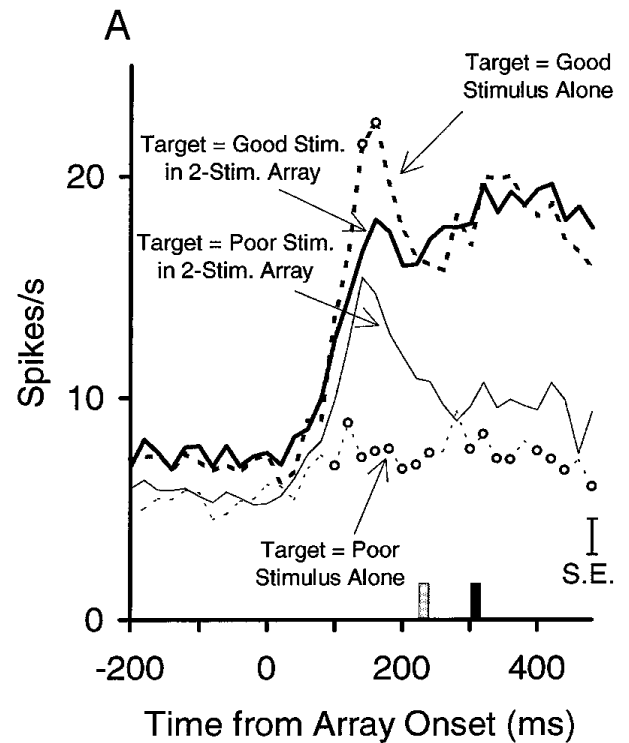

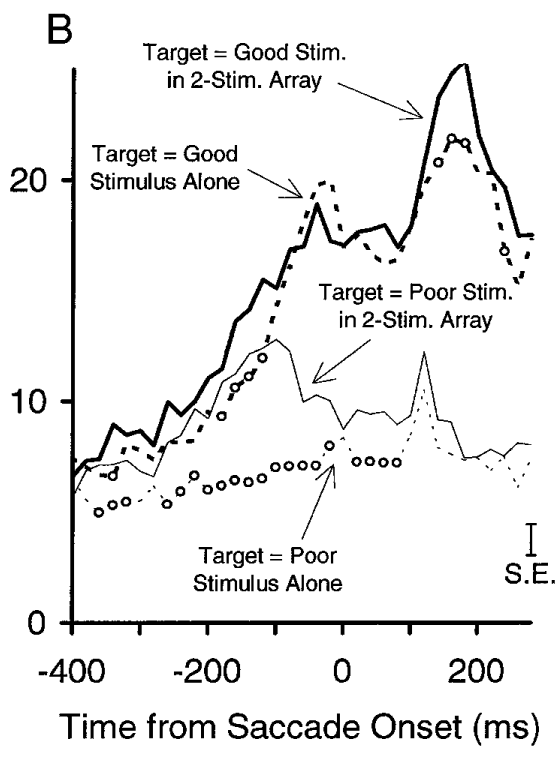

FIG. 12. Population histograms from 58 cells comparing the response to the 2stimulus array with the responses to the component stimuli presented alone. A: responses time locked to array onset. Stippled and solid vertical bars indicate average saccadic latency for 1-stimulus and 2-stimulus arrays, respectively. Empty circles on the

"Target $=$ Good Stimulus Alone" curve indicate bins in which the response differed significantly from that in the "Target = Good Stim. in 2-Stim. Array" condition. Empty circles on the "Target $=$ Poor Stimulus Alone" curve indicate bins in which the response differed significantly from that in the "Target $=$ Poor Stim. in 2-Stim. Array" condition. $B$ : same as in $A$, but responses are time locked to saccade onset. Binwidth is $20 \mathrm{~ms}$. to the poor stimulus presented alone. The influence of the good stimulus within the RF was much reduced. The influence of the good stimulus was not entirely eliminated, however, because the response to the array never equaled the response to the poor stimulus presented alone.

When these results were taken together, by $250 \mathrm{~ms}$ after array onset, the sensory interactions between the good and poor stimuli in the array were largely eliminated. Instead, cells responded to the two-stimulus array largely as though the array contained only the target stimulus.

To test these conclusions derived from the population histograms on individual cells, we computed a $t$-test on each cell's response to the array with the good stimulus as target versus the response to the good stimulus alone. The test was computed on responses in a time window $100 \mathrm{~ms}$ before saccade onset. Responses to the two-stimulus array were significantly different from those to the good stimulus alone for only 10 of 58 cells. Of these, eight cells gave better responses to the two-stimulus array than to the good stimulus alone, and only two responded less to the array than to the good stimulus alone. Thus, for nearly all cells, the inhibitory influence of the poor stimulus was eliminated when the good stimulus was the target. These effects can be observed in the scatter plot of Fig. $13 \mathrm{~A}$.

Turning to the trials with the poor stimulus as target, we computed a $t$-test for each cell on the response to the array with the poor stimulus as target versus the response to the poor stimulus alone. Again, the test was computed in a time window $100 \mathrm{~ms}$ before saccade onset. Responses to the twostimulus array and the poor stimulus alone were significantly different for only 9 of 58 cells. Of the nine, eight cells gave a smaller response to the poor stimulus alone, and one cell gave a smaller response to the array with the poor stimulus as target. Thus, for the large majority of cells, the excitatory effect of the good stimulus in the array on the cell's response was strongly reduced when the poor stimulus was the target. This effect can be observed in the scatter plot of Fig. $13 B$.

These results indicate that the effect of target selection on the response to the two-stimulus array was to drive the cell's response to a level that was similar to what it would be for either the good or the poor stimulus alone, depending on which was the target. If so, then the magnitude of the target effect for a given cell should depend at least in part on that cell's selectivity for the good and poor stimuli. To test this prediction, we compared the TEI and the stimulus selectivity index (SEI), described in previous sections. Figure 14 shows a scatter plot of the two indexes across the population of cells. As predicted, there is a clear relationship between the two measures, with a correlation coefficient $(r)$ of $0.52(P<$ $0.001)$. Likewise, there was a clear relationship between the target effect and delay activity, with a correlation coefficient $(r)$ of $0.60(P<0.001)$ between the TEI and DAI.

Search task with saccades (1- and 2-stimulus arrays): arrays across the VM

The results described in the previous sections were obtained when the search array was confined entirely to the contralateral field. However, the results changed significantly when one of the stimuli in the array was located within the contralateral field and one was in the ipsilateral field. Figure 15 shows the response, averaged across the population, to the two-stimulus array when the good versus poor stimulus was the target. Figure $15 \mathrm{~A}$ shows the results from the configuration in which the good stimulus was in the contralateral field and the poor stimulus was in the ipsilateral field, whereas $B$ shows the results from the opposite spatial configuration. Only the data from the blocked cue design are used in these analyses, because some of the comparisons described in the following sections were not run in the random cue design.

In contrast to the results with both stimuli contained within the contralateral field, there appeared to be little or no effect of target selection in the population histogram until approximately the time of saccade initiation. The saccade occurred, on average, at $256 \mathrm{~ms}$ after array onset with the good stimulus in the contralateral field and at $257 \mathrm{~ms}$ after array onset with the good stimulus in the ipsilateral field. This can be seen more clearly in Fig. 15, $C$ and $D$, which shows the same responses as in $A$ and $B$, respectively, but time locked to the initiation of the saccade. 

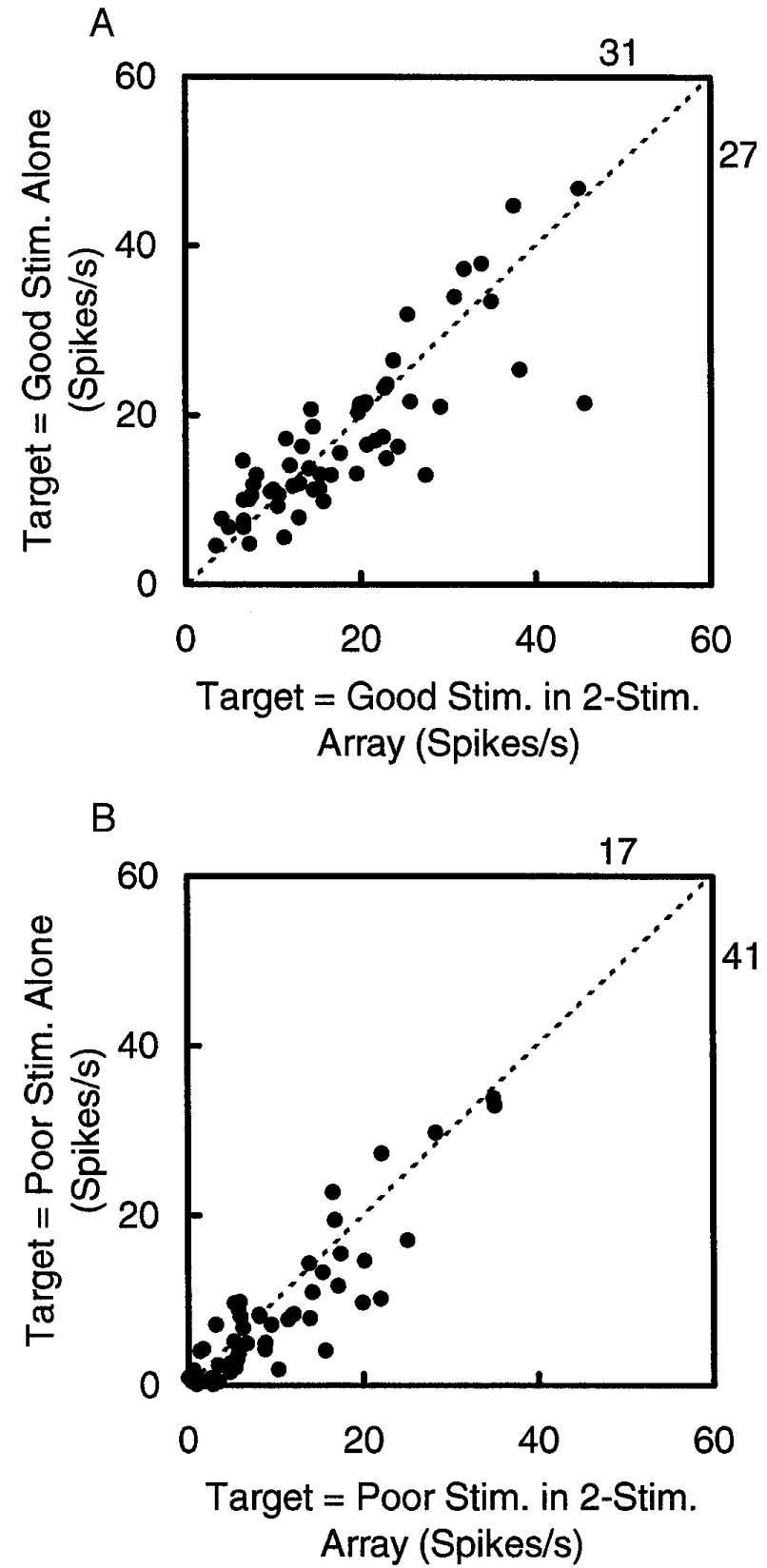

FIG. 13. Responses to the target stimulus presented alone compared with when the same target was presented in the 2-stimulus array. $A$ : responses to the good stimulus as target, measured in a time window spanning the last $100 \mathrm{~ms}$ before saccade onset. $B$ : responses to the poor stimulus as target, in the same time window as in $A$.

Because this saccadic reaction time was significantly faster than the 307-ms saccadic reaction time found with both stimuli in the contralateral field (paired $t$-test, $P<$ $0.001)$, this difference in reaction times might potentially account for the fact that the target effects failed to precede the onset of the saccade in the cross-midline but not the within-hemifield condition. Specifically, the effects of target selection on the response may have actually occurred at the same time after the onset of the array in all spatial configurations of the array; the only difference between the crossmidline and within-hemifield configurations may have been the time of the saccade. Therefore, to test this, we computed the onset of the target effect in the population histogram in the cross-midline configuration. We computed a paired $t$ test on the firing rate in the good stimulus as target versus poor stimulus as target conditions, for each $20-\mathrm{ms}$ bin in the histogram. We then determined the time at which two consecutive bins showed a significant difference in response on trials with the good stimulus as target compared with when the poor stimulus was the target. This analysis indicated that a significant target effect occurred at $320 \mathrm{~ms}$ after array onset when the good stimulus was in the contralateral field and $300 \mathrm{~ms}$ after array onset when the good stimulus was in the ipsilateral field. These times were $>100 \mathrm{~ms}$ later than the onset of significant target effects in the withinhemifield condition, which occurred 170-180 ms after array onset. Thus we could reject the hypothesis that the only difference between the target effects in the within-hemifield and cross-midline conditions was the time of the eye movement. Rather, the saccades occurred earlier and the target effects occurred later in the cross-midline condition than in the within-hemifield condition.

To confirm that the target effects in the population histogram did not precede the onset of the saccade in the crossmidline condition, we recomputed the $t$-tests on the population histograms time locked to the onset of the saccade. This analysis indicated that a significant target effect began either 120 or $80 \mathrm{~ms}$ after onset of the saccade with the good stimulus in the contralateral or ipsilateral field, respectively. These effects of target selection occurring after the saccade were presumably caused by the eye movement bringing the image of the target stimulus onto the center of gaze.

To assess the effects of target selection on the responses of individual cells, we also computed $t$-tests on the responses of individual cells to the good versus poor stimulus as target. The tests were run using both an early time window 70$170 \mathrm{~ms}$ after array onset and a late time window spanning

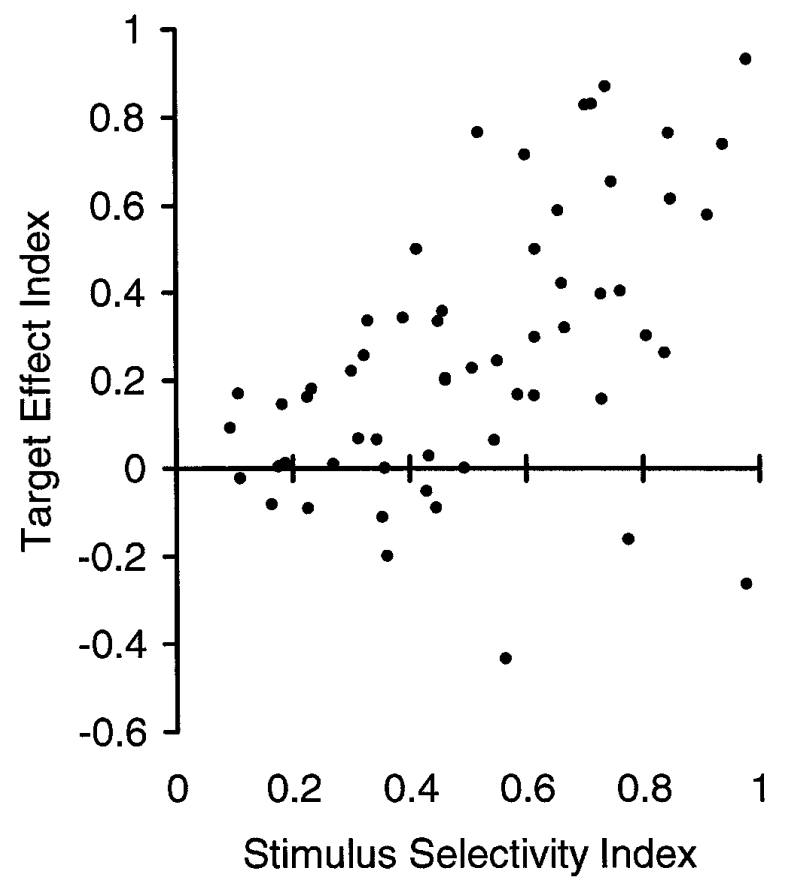

FIG. 14. Relationship between the TEI and the stimulus selectivity in- 
Good Stim. in Contra. Field
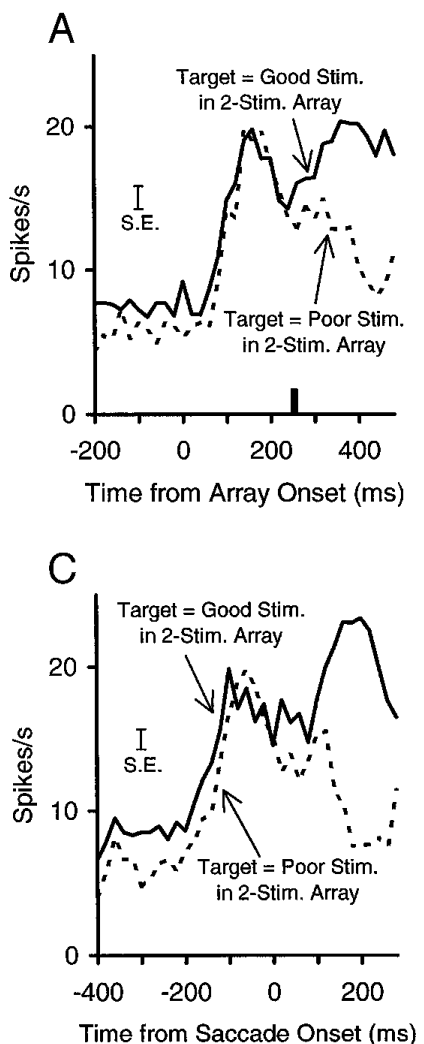

Good Stim. in Ipsi. Field

B

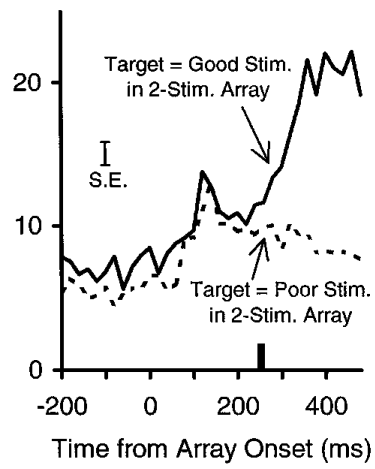

$\mathrm{D}$

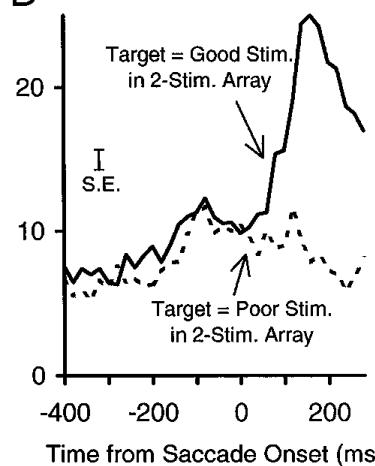

FIG. 15. Population histograms averaged from 58 cells, showing the response to the search array in which the stimuli were in opposite hemifields. In $A$ and $C$ the good stimulus in the array was positioned in the hemifield contralateral to the recording site, whereas the poor stimulus was positioned in the ipsilateral hemifield. In $B$ and $D$ the good stimulus in the array was positioned in the hemifield ipsilateral to the recording site, whereas the poor stimulus was positioned in the contralateral hemifield. In $A$ and $B$ responses are time locked to array onset (black vertical bars indicate average saccadic latency), whereas in $C$ and $D$ responses are time locked to the eye movement. Binwidth is $20 \mathrm{~ms}$.

the last $100 \mathrm{~ms}$ before the saccade on each trial. Consistent with the analysis of the population histograms, there was little or no tendency for positive target effects in either time window. In the early time window, when the good stimulus was in the contralateral field, 5/58 neurons gave a significantly larger response when the good stimulus was the target, and an equal number of cells gave a significantly smaller response. Similarly, in the late time window when the good stimulus was in the contralateral field, 6/58 neurons gave a significantly larger response when the good stimulus was the target, and an equal number of cells gave a significantly smaller response. When the good stimulus was in the ipsilateral field, the number of cells with significant target effects dropped below the number expected by chance (binomial test, $P>0.05$ ), in both the early and late windows. Thus the responses of only a few cells were significantly affected by whether the good or poor stimulus was the target in the cross-midline configuration. These effects were found when the good stimulus was in the contralateral field and were evenly split between increases and decreases in responses.

As expected, target selection had virtually no effect, on average, on the response to the good or poor stimuli pre- sented alone in the ipsilateral field, just as we found when the stimuli were located within the contralateral field.

SENSORY INTERACTIONS BETWEEN GOOD AND POOR STIMULI. Given that the target effects were so different in the crossmidline condition compared with within-hemifield, we asked whether there would be comparable differences in the sensory interactions between the two stimuli in the array. We therefore determined the sensory response to the good stimulus alone, the poor stimulus alone, and the combined good and poor stimulus in the array in the cross-midline condition. We used the responses in the target-absent trials as the best measure of sensory responses independent of target selection, just as we did when the array was confined to the contralateral field.

Figure $16 \mathrm{~A}$ shows the population histograms for the responses in the target-absent conditions when the good stimulus was in the contralateral field and the poor stimulus in the ipsilateral field. In this configuration, the response to the array was almost as large as the response to the good stimulus presented alone in the contralateral field. That is, the cells responded to the two-stimulus array as though it contained only the good stimulus in the contralateral field, with only a modest suppressive influence of the poor stimulus in the ipsilateral field. This is in contrast to the target-absent trials in the within-hemifield condition (see Fig. 10A), where the poor stimulus in the array had a substantial suppressive effect on the response to the good stimulus. In the cross-midline condition, the stimulus in the ipsilateral field has little sensory influence on the cell's response to a stimulus in the contralateral field. Putting this result together with the fact that selecting a target in the ipsilateral field had little effect on the cell's response to a contralateral stimulus suggests the following hypothesis: whichever stimulus is in the contralateral visual field largely determines the cell's response, and this dominant influence of the contralateral stimulus cannot be overcome by selecting a target stimulus in the ipsilateral visual field.

This hypothesis also fits the sensory-interaction (targetabsent) data obtained when the good stimulus was placed in the ipsilateral field and the poor stimulus was placed in the contralateral field. Figure $16 B$ shows that the population response to the two-stimulus array was nearly as small as the response to the poor stimulus presented alone in the contralateral field. Thus, in this configuration, the poor stimulus in the contralateral field largely determined the cell's response to the two-stimulus array. Again, putting this result together with our failure to find an effect of target selection in this configuration suggests that the dominant influence of the contralateral stimulus on the response to the array cannot be overcome by selecting a target in the ipsilateral field.

EFFECTS OF TARGET SELECTION ON SENSORY INTERACTIONS. The last step was to ask how, in the target-selection trials, the response to the array with the good or poor stimulus as the target compared with the response to those same stimuli presented alone. For this purpose, we compared the responses to 1 ) the array with the good stimulus as the target, 2 ) the array with the poor stimulus as the target, 3 ) the good stimulus presented alone, and 4 ) the poor stimulus presented alone. For the latter two responses, we used the data from the target-present trials, although as indicated above, the 
Good Stim. in Contra. Field

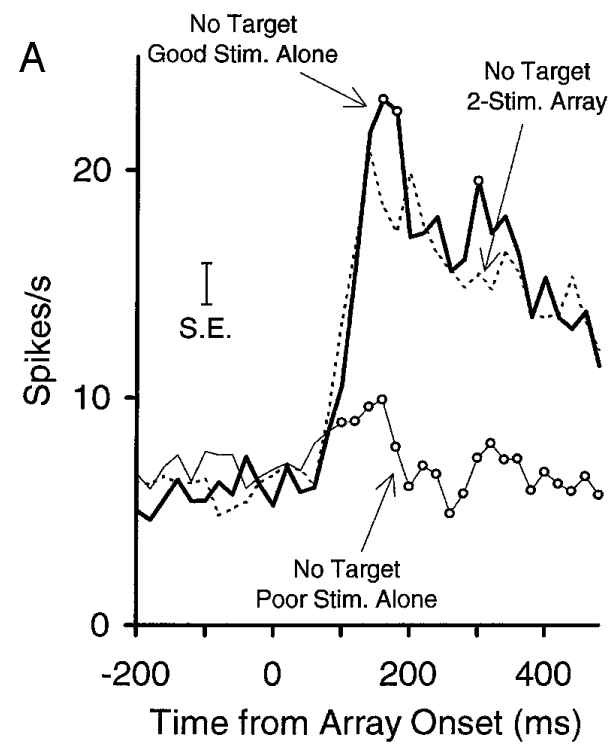

Good Stim. in Ipsi. Field

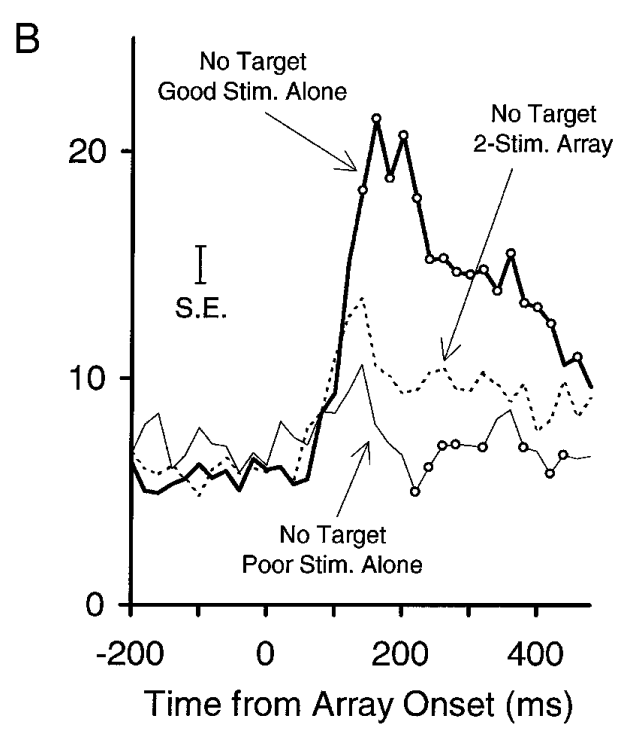

FIG. 16. Population histograms averaged from 58 cells, comparing the response to the good and poor stimuli presented alone with the response to the 2 -stimulus array. In all 3 conditions responses were measured in target-absent trials. In $A$ the good stimulus was in the contralateral hemifield and the poor stimulus was in the ipsilateral hemifield, whereas in $B$ the good stimulus was in the ipsilateral hemifield and the poor stimulus was in the contralateral hemifield. Empty circles on the "No Target, Good Stim. Alone"' curve indicate bins where the response was significantly different from that in the "No Target, 2-Stim. Array" condition. Empty circles on the "No Target, Poor Stim. Alone" curve indicate bins where the response was significantly different from that in the "No Target, 2-Stim. Array" condition. Binwidth is $20 \mathrm{~ms}$. responses on these trials were the same as on the targetabsent trials.

Figure 17 shows the population histograms for the four conditions, time locked to stimulus onset $(A$ and $B$ ) and time locked to saccade onset $(C$ and $D)$. With the good stimulus in the contralateral field ( $A$ and $C$ ), the response to the array remains much closer to the response to the good stimulus presented alone than to the response to the poor stimulus presented alone, regardless of which stimulus is the target. With the poor stimulus in the contralateral field ( $B$ and $D$ ), the response to the array moves closer to the response to the poor stimulus alone, again irrespective of which stimulus is the target. Thus, consistent with the hypothesis presented in the previous section, the stimulus in the contralateral field dominates the response to the array, irrespective of which stimulus is selected as the target.

\section{Retinal information}

Because the target effects differed according to the spatial configuration of stimuli in the array, we asked whether the cells showed any spatial selectivity in their responses to the individual stimuli presented at different retinal locations. For this analysis, we used the responses to the good stimulus presented alone, pooling target-present and target-absent trials. Responses were averaged over a 50- to 200-ms time window after stimulus onset. According to a $t$-test computed on the responses to the good stimulus in the upper contralateral quadrant versus lower contralateral quadrant, $6 / 58$ cells showed a significant difference in response at the 2 locations (which is not different from the number of significant effects expected by chance according to a binomial test, $P>0.05$ ). When we computed the same test on responses to the good stimulus in the lower contralateral quadrant versus the lower ipsilateral quadrant, $6 / 58$ cells responded significantly better with the stimulus in the contralateral field, and 4/58 cells responded significantly better with the stimulus in the ipsilateral field. Thus, although most IT neurons gave similar responses to an individual stimulus in any quadrant, a few cells seemed to convey significant information about whether the stimulus was in the contralateral or ipsilateral visual field.

We also asked whether the response to the two-stimulus array depended on the spatial configuration of the component stimuli. According to a $t$-test computed on responses to the different array configurations, pooling across target-present and target-absent trials, 10/58 cells gave a significantly different response to the array confined to the contralateral field, depending on whether the good stimulus was in the upper versus lower quadrant. In addition, 29/58 cells gave a significantly better response to the array when the good stimulus was in the contralateral field than when it was in the ipsilateral field, and 8 cells gave a significantly better response in the opposite configuration. This preference for the stimulus in the contralateral field is consistent with the fact that the contralateral stimulus had a dominant effect on the response to the array, described in the previous section.

\section{Three- and five-item search array experiment}

Under natural conditions, there will frequently be more than two objects in the visual field. To test whether the target effect would generalize to larger search arrays, 20 additional cells were studied in 1 monkey using the same general task but with search arrays consisting of 3 or 5 stimuli arranged in a hemicircle within the hemifield contralateral to the recorded hemisphere. One of the stimuli was a good stimulus for the cell, and the remainder were poor stimuli. The poor stimulus that caused the least response was treated as the single "poor stimulus" in all of the experimental manipulations and analyses to be presented below. Different cue stimuli were presented in separate blocks.

Of the 20 recorded units, 17 were significantly responsive and stimulus selective. Of these 17 cells, only 10 could be used to test for the existence of a target effect, because the remaining 7 units did not give a significant response to the peripheral arrays.

The target effect with the multiple stimulus arrays appeared to be virtually identical to the effect with two-stimu- 
Good Stim. in Contra. Field
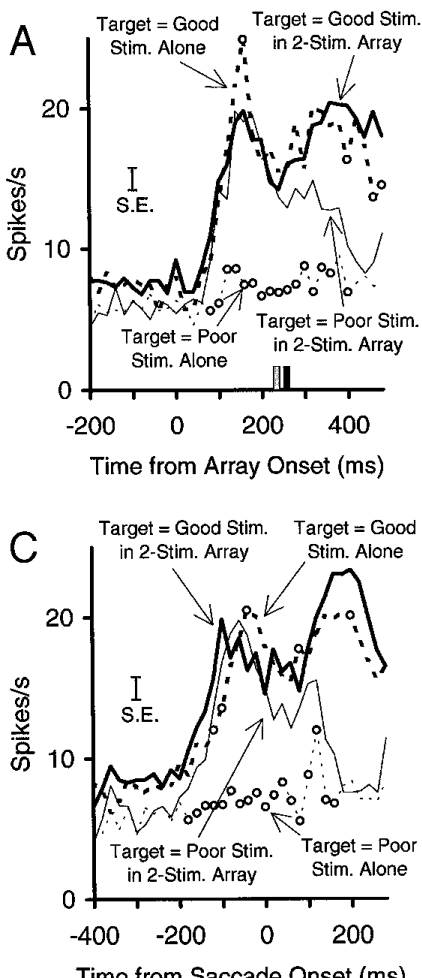

Good Stim. in Ipsi. Field
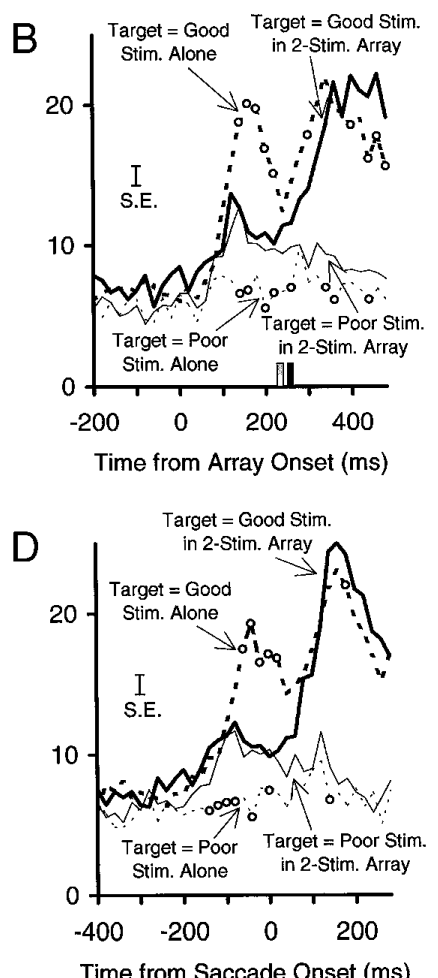

FIG. 17. Responses to the 2-stimulus array in trials with the good and poor stimuli used as targets are compared with the responses to the same stimuli presented alone in target-present trials. Population histograms are averaged from 58 cells. $A$ and $C$ : responses where the good stimulus was in the contralateral hemifield and the poor stimulus was in the ipsilateral hemifield. $B$ and $D$ : responses where the good stimulus was in the ipsilateral hemifield and the poor stimulus was in the contralateral hemifield. $A$ and $B$ : responses are time locked to array onset (stippled and solid vertical bars indicate average saccadic latency for 1-stimulus and 2-stimulus arrays, respectively). Empty circles on the "Target $=$ Good Stim. Alone" curve indicate bins where the response differed significantly from that in the " "Target = Good Stim. in 2-Stim. Array" condition. Empty circles on the "Target $=$ Poor Stim. Alone" curve indicate bins where the response differed significantly from that in the "Target $=$ Poor Stim. in 2-Stim. Array" condition. $C$ and $D$ : same as in $A$ and $B$, but responses are time locked to saccade onset. Binwidth is $20 \mathrm{~ms}$.

lus arrays in the contralateral field. Figure 18 shows the results with the 5-stimulus arrays from an example cell and the population histograms for all 10 cells tested under the same conditions. Starting $\sim 100-120 \mathrm{~ms}$ before the saccade, the firing rate stayed high on trials when the good stimulus was the target but dropped close to the baseline firing rate on trials when the poor stimulus was the target. These results are very similar to those obtained with two-stimulus arrays.

In an early time window between 70 - and 170 -ms postarray onset, only two cells showed a significant target effect (which is not different from the number of significant effects expected by chance according to a binomial test, $P>0.05$ ). Both had a higher firing rate in poor-target trials compared with good-target trials. Averaged across the 10 cells, the TEI in this time window was -0.05 . By contrast, in a later time window covering the last $100 \mathrm{~ms}$ before saccade onset, 6 cells had a significantly higher firing rate on trials with the good stimulus as target compared with the poor stimulus as target, and the mean TEI for all 10 cells was 0.28 . Thus, on average, the target effect occurred in the late phase of the response, as we found with two-stimulus arrays. The pattern of sensory interactions on target-absent trials was also very similar to what was found with two-stimulus arrays confined to the contralateral field.

In this monkey we could compare the saccadic latency to single matching stimuli and to targets in arrays of two, three, or five stimuli. The average latency increased with array size, from a minimum of $204 \mathrm{~ms}$ with a single target stimulus to a maximum of $319 \mathrm{~ms}$ with five-stimulus arrays (the slope of the best-fitting linear function was $26 \mathrm{~ms} / \mathrm{item}$; the correlation coefficient, $r$, was $0.93, P<0.001)$. The increase in latency with array size indicates that the target did not "pop out" of the array.

\section{Lever release task}

It is possible that both the cue-related activity and the target effects described in the previous sections are specific to tasks that require an eye movement to a target. To test the generality of the target effects, we measured IT responses in an additional monkey taught a version of the search task with a lever release as the behavioral response. The monkey was presented with a cue stimulus at the start of the trial. After a delay period, a search array of one to two stimuli
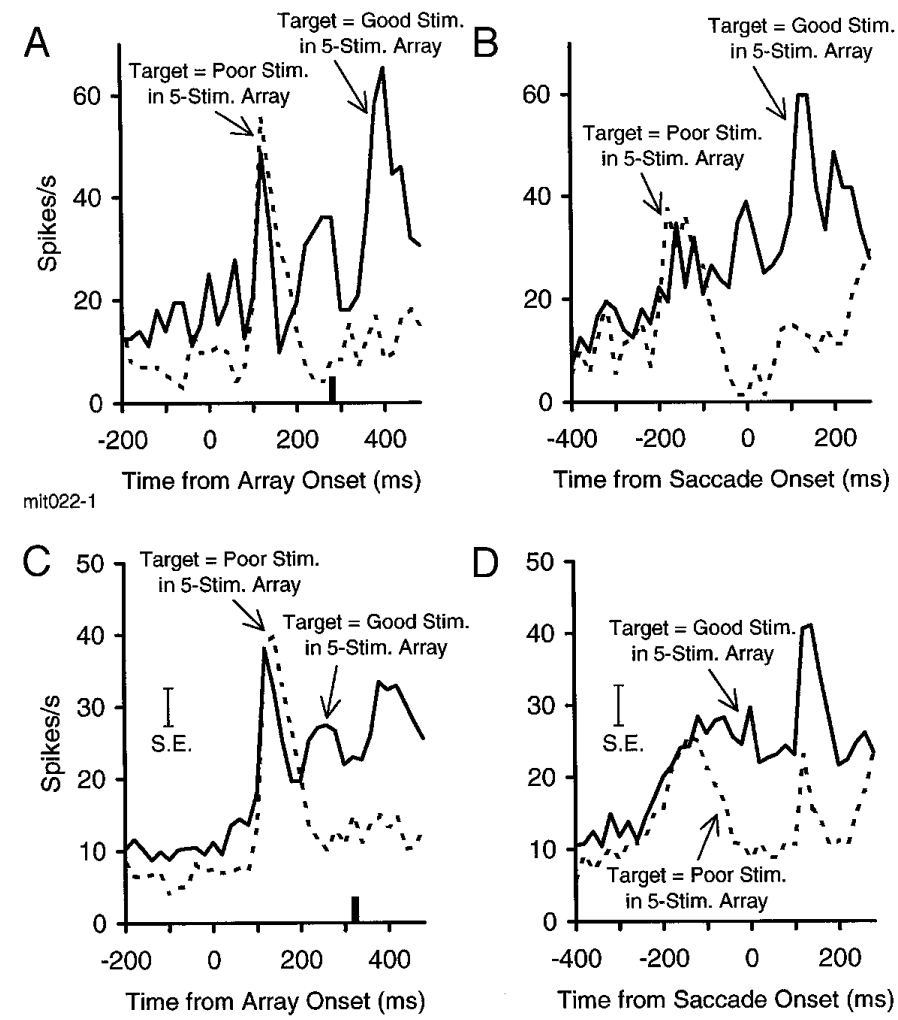

FIG. 18. $A$ and $B$ : examples of responses of an individual neuron to the 5-stimulus array. Responses are shown separately for trials in which the good stimulus was the target $(-)$ and trials in which the poor stimulus was the target $(---)$. $C$ and $D$ : same as in $A$ and $B$ but histograms are averaged from the responses of 10 neurons. In $A$ and $C$ responses are time locked to array onset (solid vertical bars indicate average saccadic latency to the target stimulus ). $B$ and $D$ : responses are time locked to the onset of the saccade. Binwidth is $20 \mathrm{~ms}$. 
appeared, and the animal was rewarded for releasing a lever if any stimulus in the array matched the previous cue (see METHODS). To facilitate comparison with the data from the saccade task, we will refer to the matching stimulus as the target and the nonmatching stimulus as the nontarget, even though the animal did not make a response directed to the location of the matching stimulus.

Of the 79 total cells recorded in 1 monkey, 14 had no significant visual response, and an additional 4 cells were not stimulus selective. The remaining 61 cells were significantly responsive and stimulus selective.

DELAY ACTIVITY. As was found for cells in the saccade task, the level of delay activity following the cue depended on which stimulus was used as the cue. Across the population of 61 selective cells, the average firing rate in the last 500 $\mathrm{ms}$ of the delay was 3.9 spikes/s following the good cue and 2.9 spikes/s following the poor cue, which was a significant difference according to a paired $t$-test $(P<0.001)$. For 28/ 61 cells that individually showed a significant difference in delay activity following the good versus poor stimulus as cue, the average firing in the delay was 3.7 versus 1.7 spikes/ s, respectively. Only 2 of 61 cells showed significantly higher activity in the delay following the poor stimulus used as a cue than following the good stimulus.

Finally, as was found in the saccade task, baseline activity in the precue period ( $300 \mathrm{~ms}$ preceding cue onset) was also different for some cells depending on the cue presented in a given block. This difference was significant for $16 / 61$ cells according to a $t$-test computed for each cell. For 14 of these cells, activity was higher in blocks with the good cue (4.7 spikes/s) than in blocks with the poor cue $(3.1$ spikes/s), whereas the opposite was true for the remaining 2 cells.

EFFECTS OF TARGET SELECTION ON TWO-STIMULUS ARRAYS. Of the 61 stimulus selective cells considered in the previous section, only 44 gave a significant response to any of the extrafoveal 2-stimulus arrays, according to a paired $t$-test computed on the pre- and poststimulus firing rates. These 44 cells are the subject of the analyses presented below.

Because the results differed depending on whether the stimuli were located in the contralateral or ipsilateral field, we will first present the results with the array in the contralateral field, with one stimulus in the upper quadrant and one in the lower quadrant. For these analyses, we pooled the data across the two different spatial configurations of the array in the contralateral field (good stimulus in upper quadrant and poor stimulus in the lower, and vice versa).

Figure 19, $A$ and $B$, shows the responses of an individual cell to the search array on trials in which the target was the good stimulus for the recorded cell versus responses on trials in which the target was the poor stimulus. Figure 19, C and $D$, shows responses to the same comparison conditions averaged across all the cells. As in the saccade task, whether the good or poor stimulus was the target made little difference in the initial response to the array. However, well before the behavioral response, responses diverged depending on which stimulus was the target. The firing rate remained high if the good stimulus was the target but was somewhat suppressed if the poor stimulus was the target. These effects are qualitatively similar to the target effects in the saccade task but appear to be smaller in magnitude.
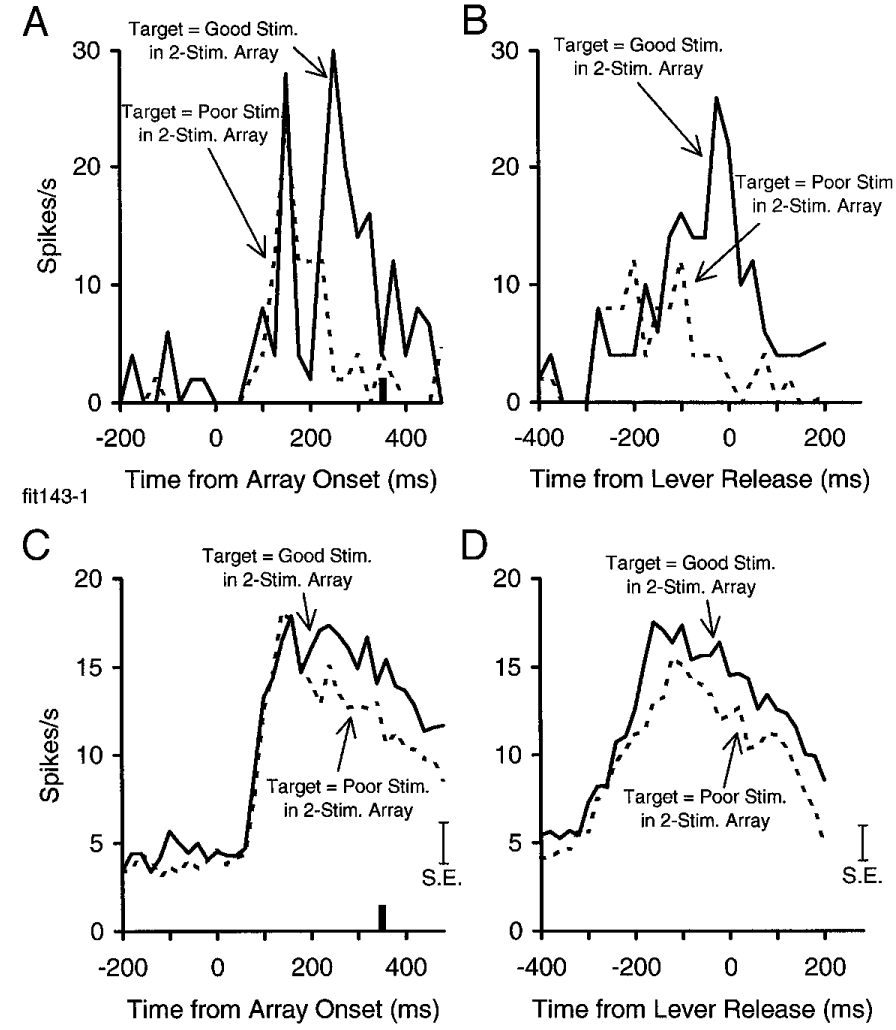

FIG. 19. Data from the lever release task. $A$ and $B$ : responses of an individual neuron to the 2-stimulus array confined to the contralateral hemifield. $C$ and $D$ : same as in $A$ and $B$ but averaged across the population of 44 neurons. In $A$ and $C$ responses are time locked to array onset (solid vertical bars indicate average latency of the lever release from array onset). $B$ and $D$ : responses are time locked to the lever release. Binwidth is $25 \mathrm{~ms}$ in $A$ and $B$, and $20 \mathrm{~ms}$ in $C$ and $D$.

To determine the time at which the population response to the good stimulus became suppressed when the poor stimulus was the target, we computed a paired $t$-test (evaluated at $P<0.05$ ) on each 20 -ms bin in the population histograms. The onset of suppression was defined to begin at the first of two consecutive bins that showed a significant difference in response on the good and poor target trials. According to this analysis, the population response to the good stimulus became significantly suppressed at $220-240 \mathrm{~ms}$ after array onset when the poor stimulus was the target. To determine the onset of the target effect relative to the behavioral response, we recomputed the histograms for both the individual cell example and the population, time locked to the lever release rather than the stimulus onset (Fig. 19, $B$ and D). According to a time series of paired $t$-tests, the population responses became significantly different $180-160 \mathrm{~ms}$ before the lever release. After the responses diverged, they remained significantly different through the time of the behavioral response except for a few interspersed individual bins.

To examine the difference between the early and late phase of the response to the array across the population of cells, we computed the TEI for an early response window of 70-170 ms postarray onset and a late response window spanning the $100 \mathrm{~ms}$ before the lever release. The population average TEI in the early time window was 0.04 , whereas that in the late time window was 0.11 , and the difference 
approached significance (paired $t$-test, $P=0.06$ ). Again, this is the same pattern of results found for the TEI in the saccade task, but the magnitude of the effects is smaller. The TEI in the late window corresponds to a $24.7 \%$ increase in firing rate when the good stimulus was the target compared with when the poor stimulus was the target, or a 53.1\% increase in the sensory evoked response with the baseline activity subtracted (averaging the baseline across trials when the good and poor stimuli were the targets). Consistent with the TEI distribution, in the late time window 9/44 cells had a significantly larger response on trials with the good stimulus as target, compared with 1 cell with a significant difference in the opposite direction, according to a $t$-test computed on the responses to the search array for each cell.

EFFECTS OF TARGET SELECTION ON ONE-STIMULUS ARRAYS. As in the saccade task, we asked whether target selection had any effect on responses when there was a single stimulus within the RF, i.e., in the absence of competition between the stimuli in the array. We therefore compared the response to the good and poor stimuli presented alone on target-present trials versus target-absent trials. This analysis showed that, as in the saccade task, there was no evidence for the type of target effects found when the good and poor stimuli were presented together in the array.

SENSORY INTERACTIONS BETWEEN GOOD AND POOR STIMULI. Because the effects of target selection were different when two stimuli competed within the RF than when there was a single stimulus, we next examined the nature of that competition. As in the saccade task, we compared the sensory response to the good stimulus alone, the poor stimulus alone, and the combined good and poor stimulus in the array in target-absent trials.

Figure 20 shows the population response histograms in the target-absent conditions. As in the saccade task, the response to the two-stimulus array was, on average, smaller than the response to the good stimulus presented alone. This is similar to the overall suppressive effect of the poor stimulus that was found in the saccade task and is evidence for a competitive interaction between the good and the poor stimulus.

EFFECTS OF TARGET SELECTION ON SENSORY INTERACTIONS. The next step was to ask how the response to the array with the good or poor stimulus as the target compared with the response to those same stimuli presented alone. For this comparison, we compared the responses to 1 ) the two-stimulus array with the good stimulus as the target, 2) the twostimulus array with the poor stimulus as the target, 3 ) the good stimulus presented alone, and 4) the poor stimulus presented alone. For the latter two responses, we used the data from the one-stimulus arrays in target-present trials, although as indicated above, the responses on these trials were about the same as on the target-absent trials.

Figure $21 A$ shows the population response histograms under these four conditions, time locked to stimulus onset, and Fig. $21 B$ shows the same responses time locked to the lever release. The histograms show that the initial population response to the two-stimulus array with the good stimulus as target was smaller than the response to the good stimulus presented alone. This is presumably due to the fact that the poor stimulus in the two-stimulus array had an overall

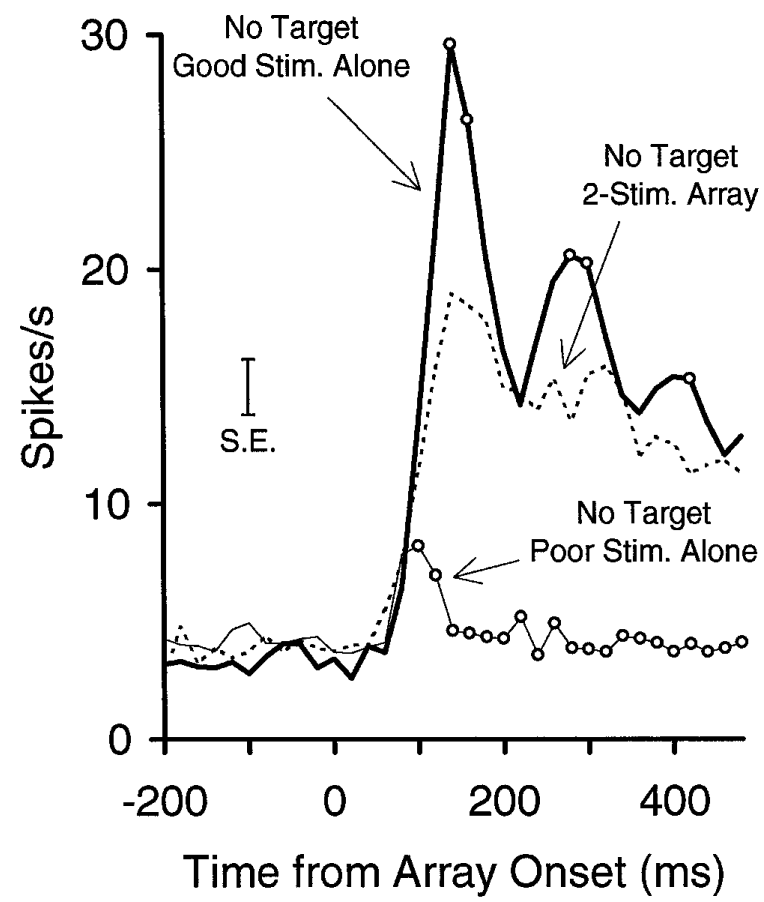

FIG. 20. Population histograms averaged from 44 cells, comparing the response to the good and poor stimuli presented alone with the response to the 2-stimulus search array. All responses were measured in target-absent trials. Empty circles on the "No Target, Good Stim. Alone" curve indicate bins where the response was significantly different from that in the "No Target, 2-Stim. Array" condition. Empty circles on the "No Target, Poor Stim. Alone" curve indicate bins where the response was significantly different from that in the "No Target, 2-Stim. Array" condition. Binwidth is $20 \mathrm{~ms}$.

inhibitory effect on the response to the good stimulus, which was shown in the previous section. However, by 150-200 $\mathrm{ms}$ after array onset, the response to the array with the good stimulus as target equaled the response to the good stimulus presented alone, i.e., the inhibitory effect of the poor stimulus was eliminated. This same trend was found when we computed a $t$-test on the responses of individual cells. In a time window from 100-200 ms after stimulus onset, 19 cells responded significantly better to the good stimulus alone than to the array with the good stimulus as target, with 1 cell showing a significant effect in the opposite direction. In a later time window, covering the last $100 \mathrm{~ms}$ before the behavioral response, only 10 cells responded better to the good stimulus alone, with 1 cell showing a significant effect in the opposite direction.

Conversely, the initial response to the two-stimulus array with the poor stimulus as target was much better than the response to the poor stimulus presented alone. This is presumably due to the fact that the cells were initially responding to the good stimulus present in the array. This initial response to the two-stimulus array began to show signs of suppression by $\sim 150-200 \mathrm{~ms}$ after array onset. That is, the influence of the good stimulus was reduced. However, the response to the array was never reduced to a level even close to that of the weak response to the poor stimulus presented alone. Again, the same result was found when a $t$-test was computed on the responses of individual cells. In the early time-window, 30 cells responded signifi- 
A

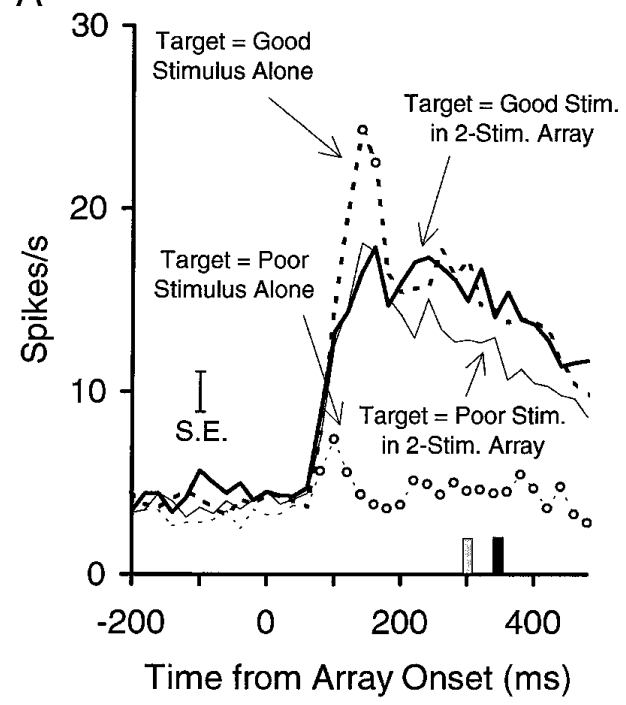

$\mathrm{B}$

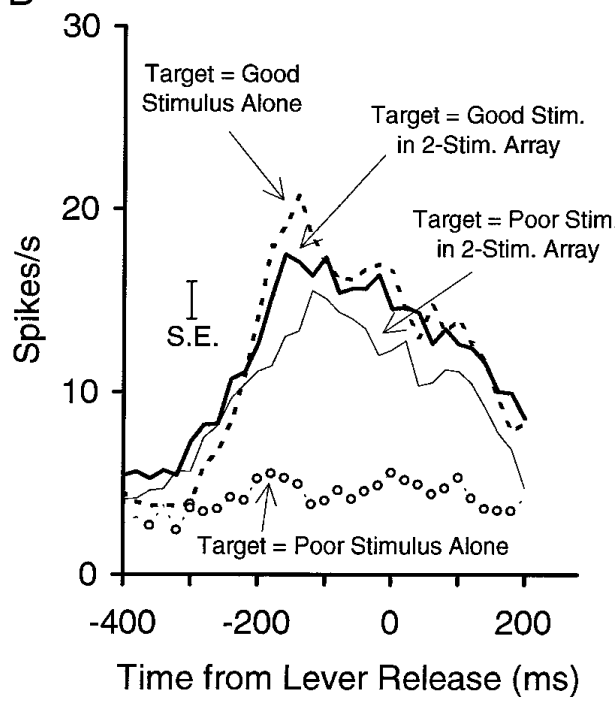

FIG. 21. Response to the 2-stimulus arrays with the good and poor stimuli as target are compared with the response to the good and poor stimuli presented alone in target-present trials. Population histograms are averaged from 44 cells. $A$ : responses time locked to array onset ( stippled and solid vertical bars indicate average latency of the lever release for 1-stimulus and 2-stimulus arrays, respectively). Empty circles on the "Target $=$ Good Stimulus Alone'" curve indicate bins where the response was significantly different from that in the "Target $=$ Good Stim. in 2-Stim. Array" condition. Empty circles on the "Target $=$ Poor Stimulus Alone", curve indicate bins where the response was significantly different from that in the "Target $=$ Poor Stim. in 2-Stim. Array" condition. $B$ : same as in $A$, but responses are time locked to the lever release. Binwidth is $20 \mathrm{~ms}$. cantly better to the 2-stimulus array than to the poor stimulus alone, and this number showed a modest drop to 24 cells in the late time window, with 1 cell showing a significant effect in the opposite direction. The results point to an asymmetry in the effects of target selection: it is apparently easier to filter out the suppressive influence of a poor stimulus than it is to filter out the excitatory influence of a good stimulus.

RESPONSES TO TWO-STIMULUS ARRAYS PRESENTED ACROSS THE MIDLINE. Positioning the two stimuli in the search array across the midline of the visual field virtually eliminated the target effect, as was found in the saccade task. Indeed, the effects of this manipulation were so similar in the saccade and lever release tasks that they will not be further described here.

\section{DIS C US S I ON}

A typical scene will contain many different objects, few of which are relevant to behavior at any given moment. Thus attentional mechanisms are needed to select relevant objects for visual processing and control over behavior. The results of the present study, taken in conjunction with the results of previous studies of attention in IT cortex, suggest that attentional selection is accomplished by suppressing the responses of IT neurons to irrelevant stimuli within their RF (Chelazzi et al. 1993; Moran and Desimone 1985). Furthermore, the detailed pattern of results in the present study are consistent with a model of attentional selection that we have termed "biased competition"' (Desimone 1996; Desimone and Duncan 1995; Duncan 1996; Luck et al. 1997).

There are two major tenets of the biased competition model. The first is that objects in the visual field compete for the responses of cells in the visual cortex. For example, if two stimuli appear simultaneously within the visual field, they will initially activate neurons in parallel throughout visual cortex. If the two stimuli are independent objects, and if a local region of cortex receives inputs from both of them, neuronal responses in that region will be determined by a competitive interaction between them. The second tenet is that these competitive interactions can be biased in favor of one stimulus or another by virtue of both "bottom-up," or stimulus-driven, influences (e.g., one stimulus has higher contrast than another) (see Reynolds and Desimone 1997; Reynolds et al. 1996) and "top-down' feedback mechanisms (e.g., one stimulus has greater behavioral relevance than another) (Luck et al. 1997; Moran and Desimone 1985 ). According to the model, these feedback mechanisms are closely associated with neural mechanisms for working memory. A computational model of attention related to this biased competition scheme has been developed by Usher and Niebur (1996). We will first consider the evidence for the biasing inputs in the present study and then consider the evidence for the competition.

\section{Bias and delay activity}

The stimulus-selective activity found during the delay period of the search task is evidence for the predicted bias in favor of the behaviorally relevant stimulus, i.e., the stimulus used as the cue and target. During the delay, most cells had a higher maintained firing rate when the good stimulus was the cue than when the poor stimulus was the cue. The change in maintained rate might come about as a result of either increased excitability or decreased inhibition.

This cue-specific maintained activity was not simply a prolonged sensory response to the cue stimulus because there was no differential delay activity following the cue when the animal was tested with the same stimulus sequence during a simple fixation task. Furthermore, this maintained activity preceded the onset of the cue when the animal could anticipate which stimulus would be used as the cue on that trial. This result was found in the blocked-cue version of the search task, in which the same cue stimulus was used for several consecutive trials. Thus no explicit stimulus was required to trigger the maintained activity before and after the cue; the only requirement was that the animal know which stimulus was the cue-target on the trial and that it be prepared to use that stimulus in the task. Because the behavioral relevance of a stimulus on a given trial depended on the animal's knowledge of the task rather than on the stimu- 
lus itself, the differential activity was presumably caused by inputs from outside the visual system.

In addition to the effects of bias on maintained activity in attentional tasks in IT cortex, several previous studies have found stimulus-specific maintained activity in shortterm memory tasks. When animals perform short-term memory tasks, such as delayed matching to sample (DMS), some IT neurons show higher activity in the delay following a preferred sample stimulus held in memory than following a nonpreferred sample (Colombo and Gross 1994; Fuster and Jervey 1981; Miller et al. 1993b; Miyashita and Chang 1988; Vogels and Orban 1994). In our view, the delay activity found in both short-term memory tasks and in visual search reflects the same underlying process. In both cases, there is a bias in favor of cells representing the features of a behaviorally relevant stimulus that is actively held in working memory. Indeed, there is little formal difference between the visual search task and the DMS task, except that the choice stimuli are distributed across space in the former task and distributed across time (typically) in the latter task. One might say that the contents of working memory guide both visual search and the selection of a matching stimulus in a top-down fashion.

As indicated above, the fact that the behavioral relevance of a stimulus is often defined by the task at hand rather than the intrinsic properties of the stimulus itself suggests that the source of the inputs that cause delay activity in IT cortex is outside the visual system per se. In favor of this idea, we recently found that delay activity in IT cortex is disrupted when the animal must process other visual stimuli during the delay (Miller et al. 1993b), whereas delay activity in prefrontal cortex is maintained under the same conditions (Miller et al. 1996). Prefrontal cortex, then, is likely to be a major source of stimulus-specific delay activity in both working memory and visual search (Fuster 1973; Fuster et al. 1982; Miller et al. 1996; Rao et al. 1997; Wilson et al. 1993).

We recently found similar spatially specific maintained activity in areas V2 and V4 when animals performed a spatial attention task (Luck et al. 1997). The animal performed a target discrimination task at one location in the visual field and ignored distracters at different locations. When the animal was cued to attend to a location within the recorded cell's RF, the cell's maintained activity increased compared with when the animal's attention was directed to a location outside the RF (Luck et al. 1997). As in the blocked-cue conditions of the present study, no physical stimulus was necessary to trigger the activity: the maintained activity started at the beginning of each trial before any stimulus was presented, as long as the animal knew that a location within the RF was the relevant one. Again, these results suggest that visual cortex receives feedback from structures involved in working memory, and this feedback biases activity in favor of those cells representing the behaviorally relevant stimulus.

\section{Resolution of competition}

If cells had linear input-output response functions, a cell's response to two stimuli, $A$ and $B$, presented simultaneously within the RF should equal the sum of the responses to $A$ and $B$ presented alone. According to the biased competition model, however, two stimuli presented within the RF should compete for the cell's response. Therefore, in the absence of any attentional bias in favor of one stimulus, the response to the pair should, on average, fall between the responses to $A$ and $B$ presented alone. If either one has a competitive advantage because it is a "stronger" sensory stimulus (e.g., because of higher contrast, etc.) (see Reynolds and Desimone 1997; Reynolds et al. 1996), the response to the pair should shift to the response to the stronger stimulus presented alone. Likewise, an attentional bias in favor of either $A$ or $B$ should have a similar effect, namely that the response to the pair should be similar to the response to the attended stimulus when it is presented alone (Luck et al. 1997; Moran and Desimone 1985). We will first consider the inhibitory interactions between the two stimuli, in the absence of any attentional bias in favor of one or the other.

SENSORY COMPETITION. The competition model predicts that two stimuli within the same RF should, on average, have a mutually suppressive interaction. This was tested in the target-absent condition, in which neither stimulus in the array was a target and, thus, there was presumably no attentional bias in favor of either one. As predicted, the response to the two-stimulus arrays in the contralateral field was, on average, intermediate between the responses to the good and poor stimuli presented alone. Consistent with this, several previous studies also found that IT responses to two stimuli presented simultaneously were less than responses to the preferred stimulus presented alone (Miller et al. 1993a; Rolls and Tovee 1995; Sato 1989). Similarly, Richmond et al. (1983) found that a spot presented at fixation had an inhibitory effect on the responses of IT cells to an extrafoveal stimulus.

The sensory interactions changed markedly when one of the two stimuli in the array was moved from the contralateral into the ipsilateral visual field. When the two stimuli were on opposite sides of the VM, neuronal responses on targetabsent trials were strongly dominated by the contralateral stimulus. The cells responded to the arrays almost as if they could not "see" the stimulus in the ipsilateral field. Yet, the ipsilateral location was clearly within the RF because cells responded well to the good stimulus presented alone when it was in the ipsilateral field. Sato $(1988,1989)$ also reported that the responses of IT neurons to two stimuli in opposite hemifields is nearly equal to the response to the contralateral stimulus alone. These results suggest that stimuli within the contralateral visual field have a very strong competitive advantage over stimuli in the ipsilateral field in IT cortex.

EFFECTS OF ATTENTION. The results revealed a powerful effect of attention on the competition between stimuli in the array, at least when the two stimuli were in the contralateral visual field. When the good stimulus was the target, the response to the array was similar to the response to the good stimulus presented alone, whereas when the poor stimulus was the target the response approached the response to the poor stimulus alone. Thus the major effect of attending to a stimulus was to eliminate the excitatory or suppressive influence of the unattended stimulus, as predicted by the biased competition model. 
In contrast to the results with two stimuli in the contralateral field, there was little or no overall effect of attention on the population response to the arrays when one stimulus was in the ipsilateral field and one was in the contralateral. In this configuration, the response to the two-stimulus array was close to the response to the contralateral stimulus presented alone, regardless of which stimulus was attended. These results are consistent with the analysis of sensory interactions in the target-absent trials, described above, in which the contralateral stimulus dominated the response to the two-stimulus array. Together, the results suggest that the stimulus in the contralateral portion of the RF has such a strong competitive advantage in IT cortex that it is difficult to overcome by any attentional bias. Consistent with this, Sato (1988) has reported that spatially directed attention to a stimulus in the ipsilateral visual field has little or no effect on the response of IT neurons to a stimulus in the contralateral field. Likewise, in a previous study of spatial attention in IT cortex (Moran and Desimone 1985), we found smaller effects of attention when competing stimuli were located in opposite hemifields than when both were located within the contralateral field (unpublished data).

Attentional effects are not always eliminated when the competing stimuli are located on opposite sides of the midline, however. We did find a few individual cells with significant attentional effects in this configuration. Furthermore, we found significant (if reduced) effects of attention in this configuration in our earlier study of spatial attention in IT cortex (J. Moran and R. Desimone, unpublished data). The magnitude of the attentional effect in this configuration may depend on task difficulty and the strength of the attentional bias.

The surprising implication of these results, however, is that the visual cortex in one hemisphere is apparently able to process stimuli in the contralateral field with little interference from stimuli in the opposite field and with correspondingly reduced need for attentional selection. Selection of a stimulus in one hemifield at the expense of the other may take place outside the traditional visual cortex, possibly in prefrontal cortex. Alternatively, selection may not take place until the final preparation for movement, such as in the frontal eye fields in a saccade task. When animals are required to select the target for an eye movement from multiple stimuli in the visual field, the responses of cells in the frontal eye field are suppressed for nontarget stimuli (Schall and Hanes 1993; Schall et al. 1995; Thompson et al. 1996). This suppression is found even when the target and nontarget stimuli are located in opposite hemifields. Even this far into the oculomotor system, however, suppression of the nontarget response is larger when the target and nontarget stimuli are located near one another rather than far apart (Schall and Hanes 1993; Schall et al. 1995).

\section{Lever release versus saccade task}

The basic effects of attention in visual search are independent of the specific motor response to the target, as we found qualitatively similar effects of attention in both the saccade and lever release versions of the task. The magnitude of the attentional effect, however, was much larger in the saccade version of the task. It is possible that the difference is due to individual differences among animals, because there were only two animals studied in the saccade task and one animal in the lever release task. Alternatively, the attentional effects may have been larger in the saccade task either because the task was more difficult or because the task required the explicit spatial localization of the target, or because of both factors. In area V4, attentional effects become larger as task difficulty is increased (Spitzer et al. 1988).

\section{Serial versus parallel processing}

A long-standing issue in the psychology of attention has been whether a target object in a complex visual display is found using serial or parallel neural mechanisms (for reviews, see Desimone and Duncan 1995; Duncan 1996; Duncan and Humphreys 1989). This issue arises when the location of the target object is not known in advance (and thus must be found on the basis of nonspatial features) and when the object is difficult to find, i.e., it does not "pop out." According to one account of serial search, the features of an object are not bound together unless the object is within the focus of spatially directed attention (Treisman and Gelade 1980; Treisman and Sato 1990; Treisman and Schmidt 1982). To find a target object defined by a conjunction of features in a search display, a roving "spotlight" of attention must be rapidly switched from object to object in the display until the target is found. The processing of objects outside the spotlight is suppressed. In this case, the search for an object based on a conjunction of features rather than its location is, nonetheless, accomplished by a spatial attention mechanism.

According to parallel processing views of visual search in psychology, target objects in a display are found by mechanisms that operate over the entire visual field at once (Atkinson et al. 1969; Bundesen 1990; Duncan 1996; Duncan and Humphreys 1989). To find a target object, an "attentional template" of the target is distributed throughout the visual field representations of the visual processing areas. Objects that match the template are allowed to pass through, whereas nonmatching objects are suppressed.

The biased competition model is a specific neural implementation of a parallel processing scheme. In this scheme, a major processing resource is the RF of cortical neurons, and objects in a scene compete for this resource in parallel. The competition is mediated by local competitive interactions among the neurons participating in the representation of all the different objects. Feedback from systems involved in working memory biases the competition in favor of cells representing the relevant object, and cells representing irrelevant objects are ultimately suppressed (see Fig. 22).

The present results are more consistent with a parallel than with a serial processing mechanism of attention. In the saccade task, it took $\sim 100 \mathrm{~ms}$ after the onset of the visual response for the population response to the nontarget to become suppressed. By contrast, we found comparable attentional effects $<30 \mathrm{~ms}$ after the onset of the visual response in a spatial attention task (Moran and Desimone, unpublished data). This 70-ms delay in the gating effects of attention during search is evidence for an initial parallel activation of object representations during search, as predicted by the biased competition model. The resolution of the competition 


\section{Cells in IT cortex}
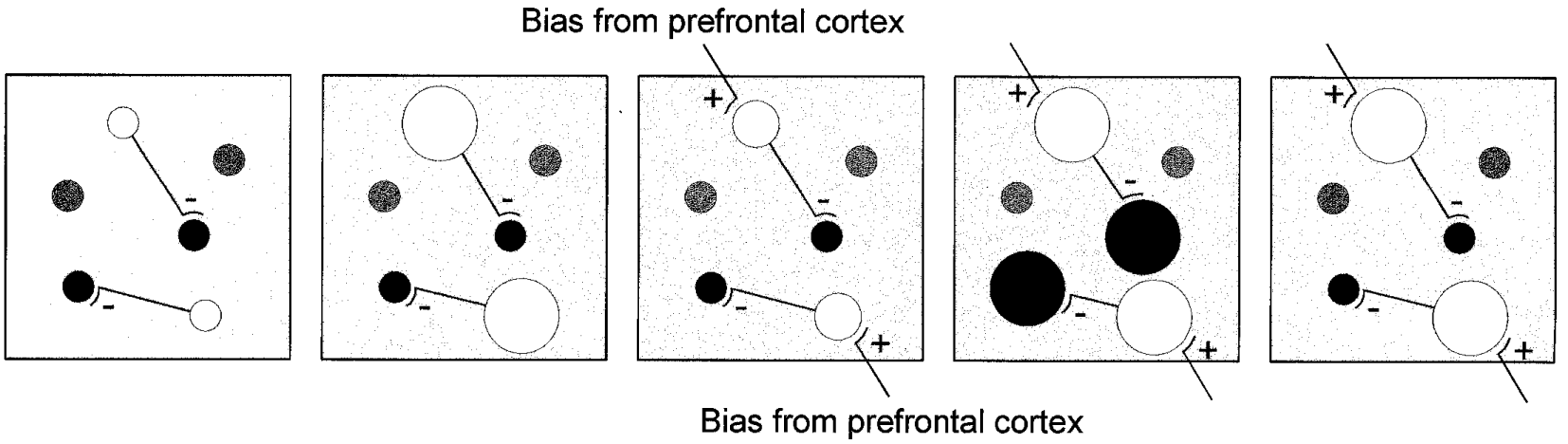

Bias from prefrontal cortex

Visual displays

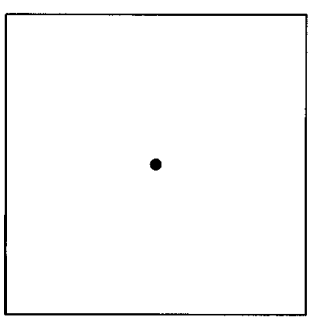

Start trial

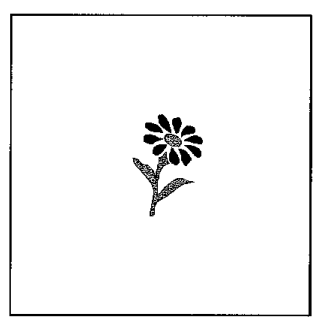

Cue

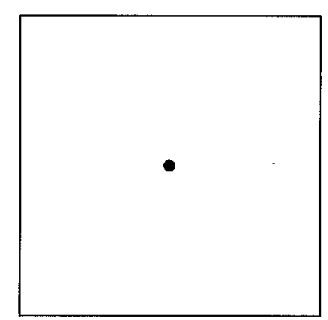

Delay

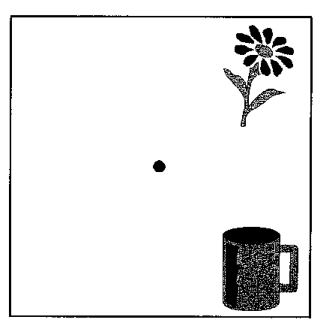

Choice (early)

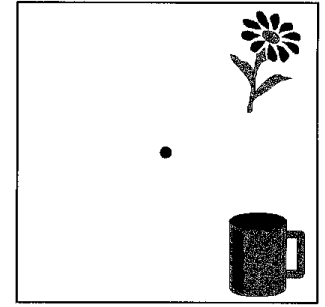

Choice (late)

FIG. 22. Schematic representation of the search task with 2-stimulus arrays confined to the contralateral hemifield, and of the pattern of activity in a representative population of inferior temporal (IT) neurons. Bottom diagrams illustrate the visual displays during the relevant portions of the task. Each dot in the top diagrams represents an individual neuron, and the size of the dot indicates relative firing rate. A specific cue (here exemplified by the flower) activates the subpopulation of IT cells tuned to any of the various features of the cue. During the delay period, this subpopulation maintains a higher level of sustained activation, relative to other cells that are tuned to the properties of the distracter. When the search array is 1st presented, both the target and the nontarget initially activate neurons for which they represent effective sensory stimuli. Later, cells tuned to the properties of the target stimulus remain active, whereas cells tuned to the properties of the distracter are suppressed. This late divergence in activation may depend on competitive interactions within IT cortex, here schematically depicted by inhibition of cells tuned to the distracter (cup) by cells tuned to the target (flower). We hypothesize that the competitive interactions are biased by top-down feedback projections from prefrontal cortex. In a given trial these projections give a competitive advantage (positive bias) to cells in IT coding the cue-target stimulus in that trial, at the expenses of cells coding the distracter.

takes longer during search for a complex object than during spatially directed attention presumably because targets and nontargets share many nonspatial features.

Additional evidence in favor of parallel processing comes from a study by Motter (1994) in area V4. Animals were presented with an array of red and green stimuli distributed over a wide extent of the central visual field. When the animals were cued to attend to stimuli of one color, cells preferring that color gave enhanced responses to the appropriately colored stimuli at any locations in the array. Apparently the feedback biasing input to cortex can target cells with the appropriate feature preference at all locations in the visual field simultaneously.

We thank J. Sewell and T. Galkin for help with the histology and J. Hart and R. Hoag for help in training the monkeys.

This research was supported in part by a grant from the Human Frontier Science Program Organization to R. Desimone and J. Duncan, by grants from Office of Naval Research (N00014-91-J-1347) and Air Force Office of Scientific Research (AFOSR-90-0043) to J. Duncan, and by a fellowship from the Human Frontier Science Program to L. Chelazzi.

Address for reprint requests: R. Desimone, Laboratory of Neuropsychol- ogy, Bldg. 49, Rm. 1B80, 49 Convent Dr., MSC 4415, Bethesda, MD 20892-4415.

Received 24 October 1997; accepted in final form 17 August 1998.

\section{REFERENCES}

Atkinson, R. C., Holmgren, J. E., And Juola, J. F. Processing time as influenced by the number of elements in a visual display. Percept. Psychophys. 6: 321-326, 1969.

Broadbent, D. E. Perception and Communication. London: Pergamon, 1958.

Bundesen, C. A theory of visual attention. Psychol. Rev. 97: 523-547, 1990.

Bushnell, M. C., Goldberg, M. E., And Robinson, D. L. Behavioral enhancement of visual responses in monkey cerebral cortex. I. Modulation in posterior parietal cortex related to selective visual attention. J. Neurophysiol. 46: 755-772, 1981.

Chelazzi, L., Miller, E. K., Duncan, J., and Desimone, R. A neural basis for visual search in inferior temporal cortex. Nature 363: 345-347, 1993.

Colby, C. L., Duhamel, J.-R., And Goldberg, M. E. Visual, presaccadic, and cognitive activation of single neurons in monkey lateral intraparietal area. J. Neurophysiol. 76: 2841-2852, 1996.

Colombo, M. AND Gross, C. G. Responses of inferior temporal cortex and 
hippocampal neurons during delayed matching to sample in monkeys (Macaca fascicularis). Behav. Neurosci. 108: 443-455, 1994.

Connor, C. E., Gallant, J. L., Preddie, D. C., and Van Essen, D. C. Responses in area V4 depend on the spatial relationship between stimulus and attention. J. Neurophysiol. 75: 1306-1308, 1996.

Desimone, R. Neural mechanisms for visual memory and their role in attention. Proc. Natl. Acad. Sci. USA 93: 13494-13499, 1996.

Desimone, R. AND DunCAN, J. Neural mechanisms of selective visual attention. Annu. Rev. Neurosci. 18: 193-222, 1995.

DUNCAN, J. Cooperating brain systems in selective perception and action. In: Attention and Performance XVI, edited by T. Inui and J. L. McClelland. Cambridge, MA: MIT Press, 1996, p. 549-578.

DunCAN, J. AND HumPhreYs, G. W. Visual search and stimulus similarity. Psychol. Rev. 96: 433-458, 1989.

FusteR, J. M. Unit activity in prefrontal cortex during delayed-response performance: neuronal correlates of transient memory. J. Neurophysiol. 36: 61-78, 1973.

Fuster, J. M., Bauer, R. H., AND Jervey, J. P. Cellular discharge in the dorsolateral prefrontal cortex of the monkey in cognitive tasks. Exp. Neurol. 77: 679-694, 1982.

Fuster, J. M. AND JERVEY, J. P. Inferotemporal neurons distinguish and retain behaviorally relevant features of visual stimuli. Science 212: $952-$ 955, 1981.

Haenny, P. E., Maunsell, J.H.R., And Schiller, P. H. State dependent activity in monkey visual cortex. II. Retinal and extraretinal factors in V4. Exp. Brain Res. 69: 245-259, 1988.

Luck, S. J., Chelazzi, L., Hillyard, S. A., and Desimone, R. Neural mechanisms of spatial selective attention in areas V1, V2 and V4 of macaque visual cortex. J. Neurophysiol. 77: 24-42, 1997.

Lynch, J. C., Mountcastle, V. B., Talbot, W. H., and Yin, T. C. Parietal lobe mechanisms for directed visual attention. J. Neurophysiol. 40: 362389, 1977.

Maunsell, J.H.R., Sclar, G., Nealey, T. A., And DePiest, D. Extraretinal representations in area V4 of the macaque monkey. Visual Neurosci. 7: $561-573,1991$.

Miller, E. K., Erickson, C. A., And Desimone, R. Neural mechanisms of visual working memory in prefrontal cortex of the macaque. J. Neurosci. 16: 5154-5167, 1996.

Miller, E. K., Gochin, P. M., AND Gross, C. G. Suppression of visual responses of neurons in inferior temporal cortex of the awake macaque monkey by addition of a second stimulus. Brain Res. 616: 25-29, 1993a.

Miller, E. K., Li, L., AND Desimone, R. Activity of neurons in anterior inferior temporal cortex during a short-term memory task. J. Neurosci. 13: 1460-1478, 1993b.

Miyashita, Y. AND Chang, H. S. Neuronal correlate of pictorial shortterm memory in the primate temporal cortex. Nature 331: 68-70, 1988.

Moran, J. AND Desimone, R. Selective attention gates visual processing in the extrastriate cortex. Science 229: 782-784, 1985.

MotTER, B. C. Focal attention produces spatially selective processing in visual cortical areas V1, V2 and V4 in the presence of competing stimuli. J. Neurophysiol. 70: 909-919, 1993.

MotTER, B. C. Neural correlates of attentive selection for color or luminance in extrastriate area V4. J. Neurosci. 14: 2178-2189, 1994.

NeIsser, U. Cognitive Psychology. New York: Appleton-Century-Crofts, 1967.

RaO, S. C., Rainer, G., AND Miller, E. K. Integration of what and where in the primate prefrontal cortex. Science 276: 821-824, 1997.

Reynolds, J., ChelazzI, L., Luck, S. J., And Desimone, R. Sensory interactions and effects of selective spatial attention in macaque area V2. Soc. Neurosci. Abstr. 20: 1054, 1994.

REYNOLDS, J. AND DESIMONE, R. Attention and contrast have similar effects on competitive interactions in macaque area V4. Soc. Neurosci. Abstr. 23: 302,1997
Reynolds, J., Nicholas, J., ChelaZZI, L., AND Desimone, R. Spatial attention protects macaque $\mathrm{V} 2$ and $\mathrm{V} 4$ cells from the influence of non-attended stimuli. Soc. Neurosci. Abstr. 21: 1759, 1995.

Reynolds, J., Pasternak, T., and Desimone, R. Attention increases contrast sensitivity of cells in macaque area V4. Soc. Neurosci. Abstr. 22: 1197, 1996.

Richmond, B. J., WuRTz, R. H., AND SATO, T. Visual responses of inferior temporal neurons in the awake rhesus monkey. J. Neurophysiol. 50: 1415-1432, 1983.

Robinson, D. A. A method of measuring eye movement using a scleral search coil in a magnetic field. IEEE Trans. Biomed. Eng. 10: 137-145, 1963.

Robinson, D. L., Bowman, E. M., and Kertzman, C. Covert orienting of attention in macaques. II. Contributions of parietal cortex. J. Neurophysiol. 74: 698-712, 1995.

Robinson, D. L., Goldberg, M. E., and Stanton, G. B. Parietal association cortex in the primate: sensory mechanisms and behavioral modulations. J. Neurophysiol. 41: 910-932, 1978.

Rolls, E. T. AND Tovee, H. J. The responses of single neurons in the temporal visual cortical areas of the macaque when more than one stimulus is present in the receptive field. Exp. Brain Res. 103: 409-420, 1995.

SATO, T. Effects of attention and stimulus interaction on visual responses of inferior temporal neurons in macaque. J. Neurophysiol. 60: 344-364, 1988.

SATO, T. Interactions of visual stimuli in the receptive fields of inferior temporal neurons in awake macaques. Exp. Brain Res. 77: 23-30, 1989.

Schall, J. D. AND Hanes, D. P. Neural basis of saccade target selection in frontal eye field during visual search. Nature 366: 467-469, 1993.

Schall, J. D., Hanes, D. P., Thompson, K. G., And King, D. J. Saccade target selection in frontal eye field of macaque. I. Visual and premovement activation. J. Neurosci. 15: 6905-6918, 1995.

Spitzer, H., Desimone, R., And Moran, J. Increased attention enhances both behavioral and neuronal performance. Science 240: 338-340, 1988.

Steinmetz, M. A., Connor, C. E., Constantinidis, C., And McLaughlin, J. R. Covert attention suppresses neuronal responses in area $7 \mathrm{a}$ of the posterior parietal cortex. J. Neurophysiol. 72: 1020-1023, 1994.

Thompson, K. G., Hanes, D. P., Bichot, N. P., and Schall, J. D. Perceptual and motor processing stages identified in the activity of macaque frontal eye field neurons during visual search. J. Neurophysiol. 76: 4040 4055, 1996.

Treisman, A. M. Strategies and models of selective attention. Psychol. Rev. 76: $282-299,1969$.

Treisman, A. M. and Gelade, G. A feature integration theory of attention. Cogn. Psychol. 12: 97-136, 1980.

Treisman, A. M. and Sato, S. Conjunction search revisited. J. Exp. Psychol. Hum. Percept. Perform. 16: 459-478, 1990.

TREISMAN, A. M. AND SCHMIDT, H. Illusory conjunctions in the perception of objects. Cogn. Psychol. 14: 107-141, 1982.

Treue, S. And Maunsell, J.H.R. Attentional modulation of visual motion processing in cortical areas MT and MST. Nature 382: 539-541, 1996.

Tsotsos, J. K. Analyzing vision at the complexity level. Behav. Brain Sci. 13: 423-469, 1990.

UsHer, M. AND NIEBUR, E. Modeling the temporal dynamics of IT neurons in visual search: a mechanism for top-down selective attention. J. Cogn. Neurosci. 8: 311-327, 1996.

Vogels, R. AND ORBAN, G. A. Activity of inferior temporal neurons during orientation discrimination with successively presented gratings. J. Neurophysiol. 71: 1428-1451, 1994.

Wilson, F.A.W., O'Scalaidhe, S. P., and Goldman-Rakic, P. S. Dissociation of object and spatial processing domains in primate prefrontal cortex. Science 260: 1955-1957, 1993.

Wolfe, J. M., Cave, K. R., And Franzel, S. L. Guided search: an alternative to the feature integration model for visual search. J. Exp. Psychol. Hum. Percept. Perform. 15: 419-433, 1989. 